\title{
THE LOEWY LENGTH OF A TENSOR PRODUCT OF MODULES OF A DIHEDRAL TWO-GROUP
}

\author{
ERIK DARPÖ AND CHRISTOPHER C. GILL
}

\begin{abstract}
While the finite-dimensional modules of the dihedral 2-groups over fields of characteristic 2 were classified over 30 years ago, very little is known about the tensor products of such modules. In this article, we compute the Loewy length of the tensor product of two modules of a dihedral two-group in characteristic 2. As an immediate consequence, we determine when such a tensor product has a projective direct summand.
\end{abstract}

\section{INTRODUCTION}

The tensor product is an invaluable and frequently used tool in the representation theory of finite groups. Given a field $K$ and a finite group $G$, the co-algebra structure of $K G$, defined by $\Delta(g)=g \otimes g$ for all $g \in G$, gives rise to a tensor product on the category $\bmod K G$ of finitedimensional $K G$-modules: $x \cdot(m \otimes n)=\Delta(x)(m \otimes n)$ for $m \in M, n \in N$ and $x \in K G$. The tensor product of two indecomposable $K G$-modules is usually not indecomposable, and the problem of determining a direct sum decomposition - the Clebsch-Gordan problem - is extremely difficult and in general not well understood.

One approach to studying the tensor product of $K G$-modules goes via the representation ring, or Green ring, $A(K G)$, which encodes the behaviour of the tensor product in its multiplicative structure. This approach was pioneered by J. A. Green in [6], who proved that the Green ring of a cyclic $p$-group is semi-simple. Much of the research on Green rings since has focussed on the question of semisimplicity, asking for which group algebras $K G$ the Green ring $A(K G)$ contains nilpotent elements. Notably, Benson and Carlson [3] provided a general method to construct nilpotent elements in Green rings, and defined an ideal $A(K G ; p)$ of $A(K G)$ (here $p=\operatorname{char} K$ ) such that the quotient $A(K G) / A(K G ; p)$ has no nilpotent elements.

The most complete results concerning direct sum decompositions of tensor products are for cyclic $p$-groups and the Klein four-group $V_{4}$. The indecomposable modules of $V_{4}$ over a field of characteristic 2 were first determined by Kronecker, and Conlon [5] computed the direct sum decompositions of tensor products of such modules. Both results are surveyed in [1]. The indecomposable modules of cyclic $p$-groups over a field of characteristic $p$ correspond to Jordan blocks with eigenvalue 1, with the tensor product of modules given by the Kronecker product of matrices. The problem of decomposing tensor products of cyclic $p$-groups has been studied by several authors $[6,11,7,9,2]$. However, all solutions to this problem that have been published so far, to our knowledge, are recursive; no closed formula for the decomposition seems to be known.

Let $k$ be a field of characteristic 2 , and $D_{4 q}$ the dihedral group of order $4 q$, where $q \geqslant 2$ is a 2-power. The indecomposable $k D_{4 q}$-modules were classified over thirty years ago by Ringel [10]. However, in contrast with the cyclic $p$-groups and $V_{4}$, the behaviour of the tensor product of $k D_{4 q}$-modules is not well understood. The Clebsch-Gordan problem for $k D_{4 q}$ remains far from being solved, and progress has been limited to some special cases. One example is the work [4] by Bessenrodt, classifying all endotrivial $k D_{4 q}$-modules, that is, modules $M$ with the property that $M^{*} \otimes M$ is a direct sum of the trival module and a projective module. Archer [1] studied the Benson-Carlson quotient $A\left(k D_{4 q}\right) / A\left(k D_{4 q} ; 2\right)$ when $k$ is algebraically closed, showing how

Key words and phrases. Dihedral group, modular representation tensor product, Loewy length, Green ring. MSC 2010: 20C20 (19A22).

The first author acknowledges support from the Swedish Research Council, Grant no 623-2009-709. 
multiplication in this quotient is related to the Auslander-Reiten quiver of $k D_{4 q}$, and realising the quotient as the integral group ring of an infinitely generated, torsion-free abelian group.

In this article, using the classification of indecomposable modules, we determine the Loewy length of the tensor product of any two finite-dimensional $k D_{4 q}$-modules. This provides an additional piece of information towards the understanding of the Green rings of the dihedral 2-groups, and gives certain bounds on which modules can occur as direct summands of such a tensor product. As an application, we determine precisely which tensor products have maximal Loewy length, that is, which tensor products have projective direct summands.

Write $D_{4 q}=\left\langle\sigma, \tau \mid \sigma^{2}=\tau^{2}=(\sigma \tau)^{2 q}=1\right\rangle$. Then

$$
k D_{4 q} \stackrel{\sim}{\rightarrow} \frac{k\langle X, Y\rangle}{\left(X^{2}, Y^{2},(X Y)^{q}+(Y X)^{q}\right)} \quad \text { via }\left\{\begin{array}{l}
\sigma \mapsto 1+X \\
\tau \mapsto 1+Y .
\end{array}\right.
$$

In particular, every $k D_{4 q}$-module is also a module of the algebra $\Lambda_{0}=k\langle X, Y\rangle /\left(X^{2}, Y^{2}\right)$ and conversely, every finite-dimensional $\Lambda_{0}$-module is a module of $k D_{4 q}$ for sufficiently large $q$. From here on, all modules are assumed to be finite dimensional. The algebras $k D_{4 q}$ are special biserial, hence the indecomposable modules are of three types: strings, bands and projectives. Below we recollect the classification of the indecomposable $k D_{4 q}$-modules, due to Ringel [10].

Let $\mathcal{W}$ be the set of words $a_{1} \cdots a_{n}(n \geqslant 0)$, in the alphabet $X, X^{-1}, Y, Y^{-1}$ with the property that if $a_{i} \in\left\{X, X^{-1}\right\}$ then $a_{i+1} \in\left\{Y, Y^{-1}\right\}$ and if $a_{i} \in\left\{Y, Y^{-1}\right\}$ then $a_{i+1} \in\left\{X, X^{-1}\right\}$. The empty word is denoted by 1 . For any word $w=a_{1} \cdots a_{n} \in \mathcal{W}$, set $w^{-1}=a_{n}^{-1} \cdots a_{1}^{-1}$. Take $\sim_{1}$ to be the equivalence relation on $\mathcal{W}$ identifying every word $w$ with its inverse $w^{-1}$.

Let $\mathcal{W}^{\prime} \subset \mathcal{W}$ be the set of words $w$ with the following properties:

1. $w$ has even, positive length,

2. $w$ is not a power of a word of smaller length,

3. $w$ contains letters from both $\{X, Y\}$ and $\left\{X^{-1}, Y^{-1}\right\}$.

Define an equivalence relation $\sim_{2}$ on $\mathcal{W}^{\prime}$ by saying that $w \sim_{2} w^{\prime}$ if, and only if, either $w$ or $w^{-1}$ is a cyclic permutation of $w^{\prime}$.

Given a word $w=a_{1} \cdots a_{m} \in \mathcal{W}$, a $\Lambda_{0}$-module $M(w)$ is defined as follows: $M(w)=\bigoplus_{i=0}^{m} k e_{i}$, and the action of $Z \in\{X, Y\}$ on $M(w)$ is defined by

$$
Z \cdot e_{i}= \begin{cases}e_{i-1} & \text { if } \quad i>0, a_{i}=Z \\ e_{i+1} & \text { if } i<m, a_{i+1}=Z^{-1} \\ 0 & \text { otherwise. }\end{cases}
$$

It is often helpful to picture the module $M(w)$, where $w=l_{1} l_{2} \cdots l_{m}$, by a schema

$$
k e_{0} \leftarrow_{l_{1}} k e_{1} \underset{l_{2}}{\longleftarrow} k e_{2} \underset{l_{3}}{\longleftarrow} \cdots \leftarrow_{l_{m-1}}^{\leftarrow} k e_{m-1} \underset{l_{m}}{\longleftarrow} k e_{m}
$$

The practice is to change the direction of the arrows in the schema that represent inverted elements: $l_{i}=X^{-1}$ or $l_{i}=Y^{-1}$. For example, if $w=X Y X Y^{-1} X^{-1} Y X^{-1} Y^{-1}$, then the schema of $M(w)$ is written as

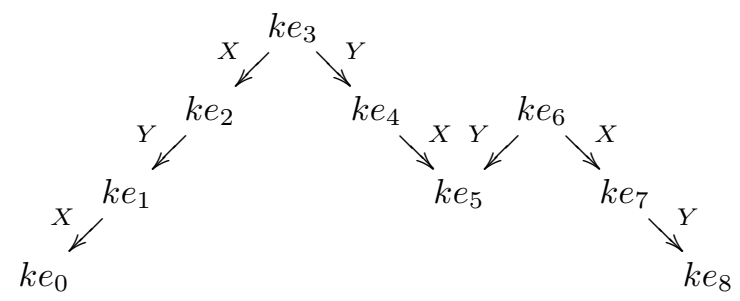

Next, let $\varphi$ be an indecomposable linear automorphism of $k^{n}$, and $w=a_{1} \cdots a_{m} \in \mathcal{W}^{\prime}$. Define $M(w, \varphi)=\bigoplus_{i \in\{0, \ldots, m-1\}} V_{i}$, where $V_{i}=\bigoplus_{j \in\{0, \ldots, n-1\}} k e_{j}^{(i)} \simeq k^{n}$ for all $i$. Denote by $T_{\varphi}$ : 
$M(w, \varphi) \rightarrow M(w, \varphi)$ the linear automorphism given by

$$
T_{\varphi}\left(e_{j}^{(i)}\right)= \begin{cases}e_{j}^{(i-1)} & \text { if } i \geqslant 2 \\ \varphi\left(e_{j}^{(i-1)}\right) & \text { if } \quad i=1 \\ e_{j}^{(m)} & \text { if } i=0\end{cases}
$$

where, for each $i$, the map $\varphi$ is viewed as a map $V_{i} \rightarrow V_{i}$ in the natural way. Now set

$$
Z \cdot e_{j}^{(i)}= \begin{cases}T_{\varphi}\left(e_{j}^{(i)}\right) & \text { if } \quad a_{i}=Z \\ T_{\varphi}^{-1}\left(e_{j}^{(i)}\right) & \text { if } \quad a_{i+1}=Z^{-1} \\ 0 & \text { otherwise. }\end{cases}
$$

This defines a $\Lambda_{0}$-modules structure on $M(w, \varphi)$. Such a module can be illustrated by a schema in the following way:

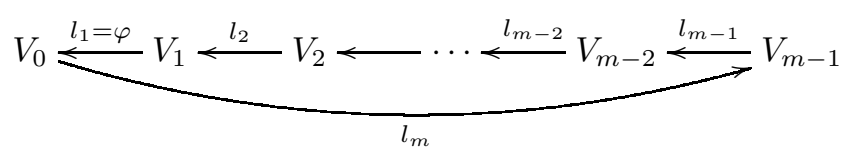

If $w=X Y X Y^{-1} X^{-1} Y X^{-1} Y^{-1}$ then $M(w, \varphi)$ is given by the following schema:

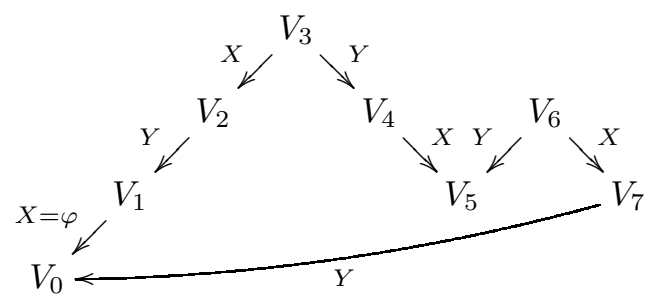

Modules isomorphic to $M(w), w \in \mathcal{W}$ are called string modules, while the ones of the type $M(w, \varphi)$ for $w \in \mathcal{W}^{\prime}$ and $\varphi$ an indecomposable linear automorphism of $k^{n}$, are called band modules.

Now, by Ringel's result, every indecomposable $\Lambda_{0}$-module is isomorphic to either a string module or a band module. The two classes are mutually disjoint. Moreover, $M(w) \simeq M\left(w^{\prime}\right)$ if, and only if, $w \sim_{1} w^{\prime}$, and $M(w, \varphi) \simeq M\left(w^{\prime}, \varphi^{\prime}\right)$ if, and only if, $w \sim_{2} w^{\prime}$ and $\varphi^{\prime}=\psi \varphi \psi^{-1}$ for some $\psi \in \operatorname{Aut}_{k}\left(k^{n}\right)$.

A $\Lambda_{0}$-module $M$ is in $\bmod \left(k D_{4 q}\right)$ if, and only if, $\left((X Y)^{q}+(Y X)^{q}\right) \cdot M=0$. This is clearly the case whenever the Loewy length of $M$ is strictly less than $2 q+1$, moreover, the regular module $k D_{4 q} k D_{4 q} \simeq M\left((X Y)^{q}\left(X^{-1} Y^{-1}\right)^{q}, 1_{k}\right)$ is the unique indecomposable projective, and the unique $k D_{4 q}$-module with Loewy length equal to $2 q+1$.

Throughout this article, the following notation and terminology is used. The least natural number is 0 . Given a non-negative real number $x,\lfloor x\rfloor$ denotes the integral part of $x$, i.e., $\lfloor x\rfloor=$ $\max \{n \in \mathbb{N} \mid n \leqslant x\}$. Let $n \in \mathbb{N}$. The $i$ th term in the binary expansion of $n$ is denoted by $[n]_{i}$, so $n=\sum_{i}[n]_{i} 2^{i}$. Further, $\nu(n)=\min \left\{i \in \mathbb{N} \mid[n]_{j}=0, \forall j<i\right\}=\max \left\{i \in \mathbb{N}\left|2^{i}\right| n\right\}$. Given $l, m \in \mathbb{N}$, we write $l \perp m$ to indicate that the binary expansions of $l$ and $m$ are disjoint, that is, $[l]_{i}+[m]_{i} \leqslant 1$ for all $i \in \mathbb{N}$. All congruences appearing are modulo two, so $l \equiv m$ always means $2 \mid(l-m)$. By $\delta_{i, j}$ we denote the Kronecker delta. By a directed subword of a word $w \in \mathcal{W}$ we mean a word $w^{\prime}$ in either the letters $\{X, Y\}$ or $\left\{X^{-1}, Y^{-1}\right\}$ such that $w=w_{1} w^{\prime} w_{2}$ for some words $w_{1}, w_{2} \in \mathcal{W}$. A directed component of $w$ is a maximal directed subword. Clearly, every word in $\mathcal{W}$ can written in a unique way as a product of its directed components. Moreover, for every word $w \in \mathcal{W}^{\prime}$ there exists a word $w^{\prime} \in \mathcal{W}^{\prime}$ with an even number of directed components such that $w \sim_{2} w^{\prime}$, and the directed components of $w^{\prime}$ are uniquely determined by $w$. Define words

$$
A_{0}=B_{0}=1 \text {, and } A_{t+1}=B_{t} Y, \quad B_{t+1}=A_{t} X \quad \text { for all } t \in \mathbb{N} \text {. }
$$

Then, for every directed word $w \in \mathcal{W}$ of length $t$, either $w \sim_{1} A_{t}$ or $w \sim_{1} B_{t}$ holds.

A basis of a $k D_{4 q}$-module $M$ on which the algebra acts according to either of the formulae (2) and (3) is called a standard basis of $M$. If $\mathcal{B}_{M}$ and $\mathcal{B}_{N}$ are standard bases of modules $M$ 
respectively $N$, the basis $\mathcal{B}_{M} \otimes \mathcal{B}_{N}=\left\{a \otimes b \mid a \in \mathcal{B}_{M}, b \in \mathcal{B}_{N}\right\}$ of $M \otimes N$ is called a homogeneous basis. We say that an element $a \otimes b$ in a homogeneous basis is pure if it is annihilated by either $X$ or $Y$, and impure otherwise. By a subquotient of a module $M$ we mean a quotient of a submodule of $M$, equivalently, a submodule of a quotient of $M$. The top of a module $M$ is the quotient module $M / \operatorname{rad} M$, the socle, $\operatorname{soc} M \subset M$, is the maximal semisimple submodule.

The Loewy length of a module $M$ is denoted by $\ell(M)$. It is the common length of the radical series and the socle series of $M$, so it may be computed as $\ell(M)=\min \left\{n \in \mathbb{N} \mid \operatorname{rad}^{n}\left(k D_{4 q}\right) \cdot M=\right.$ $0\}$. We repeatedly make use of the fact that if $M, N$ are $k D_{4 q}$-modules, and $M^{\prime}$ is a subquotient of $M$, then $M^{\prime} \otimes N$ is a subquotient of $M \otimes N$ and hence $\ell\left(M^{\prime} \otimes N\right) \leq \ell(M \otimes N)$. The Loewy length of a string module $M(w)$ is $\ell(M)=h+1$, where $h$ denotes the maximal length of all directed subwords of $w$. For band modules, we have $\ell(M(w, \varphi))=h^{\prime}+1$, where $h^{\prime}$ is the maximal length of all directed subwords of any cyclic permutation of $w$. For computational purposes, the numbers $h$ respectively $h^{\prime}$ are often easier to work with than with the Loewy length, therefore we define $h(M)=\ell(M)-1$ for $M \in \bmod \left(k D_{4 q}\right)$. If $M$ is a module and $x \in M$, we write $\ell(x)=\ell(\langle m\rangle)$ and similarly $h(x)=h(\langle x\rangle)$.

A result which is crucial for the computational parts of this article is Lucas' theorem [8] (see also Exercise 6(a) in Chapter 1 of Stanley's book [12]). Stated below for the special case of $p=2$, it will be used throughout the text without further reference.

Theorem 1 (Lucas' theorem). For all natural numbers $r$ and $s$, the congruence relation

$$
\left(\begin{array}{l}
r \\
s
\end{array}\right) \equiv \prod_{i \in \mathbb{N}}\left(\begin{array}{l}
{[r]_{i}} \\
{[s]_{i}}
\end{array}\right)
$$

holds.

The layout of this article is as follows. In Section 2, we state the main results, giving explicit, closed formulae for the Loewy length of the tensor product of any two modules in the classifying list. The first result, Proposition 4, reduces the problem of determining the Loewy length of a tensor product $M \otimes N$ to the case when $M$ and $N$ both have simple top and simple socle. This works for modules for arbitrary finite groups. In Proposition 5 we refine the result in the case of dihedral 2-groups, showing that if $M$ and $N$ are indecomposable, and $M$ does not have simple top and simple socle, then the Loewy length of $M \otimes N$ is the maximum of the Loewy lengths of $M_{i} \otimes N$ where the $M_{i}$ are uniserial string modules corresponding to the directed components of the word defining $M$. This reduces the calculation of the Loewy length of any tensor product of modules for $D_{4 q}$ to determining the Loewy lengths of tensor products of some explicitly defined modules with simple top and simple socle. Thereafter, formulae for the Loewy length of a tensor product of such modules are given in Theorem 8. The proof of Propositions 4 and 5 are relatively short, and given in a few steps in Section 2. As for Theorem 8, its proof occupies the remaining part of the article. Sections 3-5 treat tensor products of string modules, while in Section 6, the Loewy lengths of products involving band modules are computed. The basic setup of the problem for string modules is given in Section 3. In Section 4 we prove an important auxiliary result, Proposition 14, which paves the way for proof of Theorem 8:1 in Section 5. Finally, the formulae involving bands with simple top and simple socle are proved in Section 6, mainly using the result for string modules from Section 5 .

\section{REsults}

Let $\Lambda$ be an algebra over a field $K$, and $M$ a $\Lambda$-module. Denote by $\pi: M \rightarrow M / \operatorname{rad} M$ the canonical projection. Since the top of any module is semi-simple, there exists a basis $\tilde{\mathcal{B}}$ of $M / \operatorname{rad} M$ such that $\langle\bar{b}\rangle \subset M / \operatorname{rad} M$ is simple for each $\bar{b} \in \tilde{\mathcal{B}}$. Choose a set $\mathcal{B}^{\prime} \subseteq M$ of coset representatives for the elements in $\tilde{\mathcal{B}}$; these are now a set of linearly independent in $M$, and $M=\left\langle\mathcal{B}^{\prime}\right\rangle=\sum_{b \in \mathcal{B}^{\prime}}\langle b\rangle$. Moreover, each of the submodules $\langle b\rangle, b \in \mathcal{B}^{\prime}$, has simple top $\langle\pi(b)\rangle$. Now $\mathcal{B}^{\prime}$ can be extended to a basis $\mathcal{B}$ of $M$ such that each $b \in \mathcal{B}$ is contained in $\left\langle b^{\prime}\right\rangle$ for some $b^{\prime} \in \mathcal{B}^{\prime}$. The basis $\mathcal{B}$ now has the following properties: 
1. it contains a subset $\mathcal{B}^{\prime}$ such that the elements $\pi(b), b \in \mathcal{B}^{\prime}$ form a basis of $M / \operatorname{rad} M$, each element of which generates a simple submodule;

2. each element of $\mathcal{B}$ is contained in $\left\langle b^{\prime}\right\rangle$ for some $b^{\prime} \in \mathcal{B}^{\prime}$.

A basis satisfying these properties shall be called a good basis of $M$. A subset $\mathcal{B}^{\prime}$ of $M$ satisfying the first condition is called a top basis of $M$. The preceding construction shows that good bases always exist, and that every top basis can be extended to a good basis.

Lemma 2. Every $\Lambda$-module has a good basis.

Observe that in a $k D_{4 q}$-module, a standard basis is a good basis, while a homogeneous basis of a tensor product $M \otimes N$ in general is not.

Lemma 3. If $\Lambda$ is an Artin algebra, $M \in \bmod (\Lambda)$ and $X \subset M$ a set of generators of $M$, then

$$
\ell(M)=\max _{x \in X} \ell(x) \text {. }
$$

Proof. Let $r=\max _{x \in X} \ell(x)$. The inequality $\ell(M) \geqslant r$ is immediate. On the other hand, any element $m \in M$ can be written as $m=\sum_{x \in X} m_{x}$ for some $m_{x} \in\langle x\rangle$, so $\left(\operatorname{rad}^{r} \Lambda\right) m \subset$ $\sum_{x \in X}\left(\operatorname{rad}^{r} \Lambda\right) m_{x}=0$. Hence $r \leqslant \ell(M)$.

Let $M$ and $N$ be modules, and let $\mathcal{B}_{M} \subseteq M$ and $\mathcal{B}_{N} \subseteq N$ be good bases, with top bases $\mathcal{B}_{M}^{\prime} \subseteq$ $\mathcal{B}_{M}$ and $\mathcal{B}_{N}^{\prime} \subseteq \mathcal{B}_{N}$ respectively. By Lemma $3, \ell(M \otimes N)=\max \left\{\ell(\langle a \otimes b\rangle) \mid(a, b) \in \mathcal{B}_{M} \times \mathcal{B}_{N}\right\}$. However, $\langle a \otimes b\rangle \subseteq\langle a\rangle \otimes\langle b\rangle \subseteq\left\langle a^{\prime}\right\rangle \otimes\left\langle b^{\prime}\right\rangle \subseteq M \otimes N$ for some $a^{\prime} \in \mathcal{B}_{M}^{\prime}, b^{\prime} \in \mathcal{B}_{N}^{\prime}$, so

$$
\begin{aligned}
\ell(M \otimes N) & =\max \left\{\ell(\langle a \otimes b\rangle) \mid(a, b) \in \mathcal{B}_{M} \times \mathcal{B}_{N}\right\} \leqslant \max \left\{\ell(\langle a\rangle \otimes\langle b\rangle) \mid(a, b) \in \mathcal{B}_{M} \times \mathcal{B}_{N}\right\} \\
& \leqslant \max \left\{\ell\left(\left\langle a^{\prime}\right\rangle \otimes\left\langle b^{\prime}\right\rangle\right) \mid\left(a^{\prime}, b^{\prime}\right) \in \mathcal{B}_{M}^{\prime} \times \mathcal{B}_{N}^{\prime}\right\} \leqslant \ell(M \otimes N)
\end{aligned}
$$

i.e.,

$$
\ell(M \otimes N)=\max \left\{\ell\left(\left\langle a^{\prime}\right\rangle \otimes\left\langle b^{\prime}\right\rangle\right) \mid\left(a^{\prime}, b^{\prime}\right) \in \mathcal{B}_{M}^{\prime} \times \mathcal{B}_{N}^{\prime}\right\} .
$$

Proposition 4. If $K$ is a field, $G$ a finite group and $M, N \in \bmod K G$, then $\ell(M \otimes N)$ is the maximum of $\ell(A \otimes B)$ where $A, B$ are subquotients of $M$ and $N$ respectively with simple top and simple socle.

Proof. By the preceding discussion and Lemma 2, we may assume $M$ and $N$ have simple top. On the other hand, $\ell(M \otimes N)=\ell\left((M \otimes N)^{*}\right)=\ell\left(M^{*} \otimes N^{*}\right)$ and, again by applying the argument preceding this lemma, $\ell\left(M^{*} \otimes N^{*}\right)$ is the maximum of $\ell(U \otimes V)$, where $U \subset M^{*}, V \subset N^{*}$ run though all submodules with simple top. Since $M^{*}, N^{*}$ have simple socle, so have $U, V$. Now $\ell(U \otimes V)=\ell\left(U^{*} \otimes V^{*}\right)$, and $U^{*}$ and $V^{*}$ are subquotients of $M$ and $N$ respectively, with simple top and simple socle.

The $\Lambda_{0}$-modules with simple top and simple socle are precisely the uniserial string modules, that is, those isomorphic to $M\left(A_{t}\right), M\left(B_{t}\right)$ for $t \in \mathbb{N}$, and the band modules isomorphic to $M\left(A_{l} B_{m}^{-1}, \rho\right)$ for $l, m \in \mathbb{N}, \rho \in k \backslash\{0\}$.

While Proposition 4 is valid for all finite group algebras, we can do slightly better with $k D_{4 q}$.

Proposition 5. Let $N \in \bmod \left(k D_{4 q}\right)$.

1. If $w \in \mathcal{W}$ is a word with directed components $w_{i}, i \in\{1, \ldots, m\}$, and $M=M(w)$, then

$$
\ell(M \otimes N)=\max \left\{\ell\left(M\left(w_{i}\right) \otimes N\right) \mid i \in\{1, \ldots, m\}\right\} .
$$

2. Let $m$ and $n$ be positive integers, $w \in \mathcal{W}^{\prime}$ is a word with directed components $w_{i}, i \in$ $\{1, \ldots, 2 m\}, \varphi$ an indecomposable automorphism of $k^{n}$, and $M=M(w, \varphi)$. If $m$ and $n$ are not both equal to 1 , then

$$
\ell(M(w, \varphi) \otimes N)=\max \left\{\ell\left(M\left(w_{i}\right) \otimes N\right) \mid i \in\{1, \ldots, 2 m\}\right\} .
$$

Note that if $m=n=1$ in the second statement above, then $M$ itself has simple top and simple socle. 
Proof. In both cases of the proposition, under the respective assumptions, the modules $M\left(w_{i}\right)$ are subquotients of $M$. Hence $\ell(M \otimes N) \geqslant \max \left\{M\left(w_{i}\right) \otimes N\right\}_{i}$. On the other hand, $M$ itself is a subquotient, in the first case of $\bigoplus_{i} M\left(w_{i}\right)$, and in the second case of $\left(\bigoplus_{i} M\left(w_{i}\right)\right)^{\oplus n}$. Either way, it follows that $\ell(M \otimes N) \leqslant \max \left\{\ell\left(M\left(w_{i}\right) \otimes N\right)\right\}_{i}$, proving the assertion.

Remark 6 . Let $G$ be a $p$-group and $K$ a field of characteristic $p$. Then the regular module ${ }_{K G} K G$ is indecomposable, and a $M \in \bmod K G$ contains a projective direct summand precisely when $\ell(M)=\ell\left({ }_{K G} K G\right)$. From Proposition 4 follows that for $M, N \in \bmod K G$, the tensor product $M \otimes N$ has a projective direct summand if, and only if, there exist subquotients $A$ and $B$, of $M$ and $N$ respectively, with simple top and simple socle, such that $A \otimes B$ has a projective direct summand. In the case of $K G=k D_{4 q}$, Proposition 5 specifies precisely which subquotients $A$ and $B$ need to be considered.

Given $l, m \in \mathbb{N}$, let $s \in \mathbb{N}$ be the smallest number such that $[l]_{r}+[m]_{r} \leqslant 1$ for all $r \geqslant s$, and $\lambda=\sum_{i \geqslant s}[l]_{i}, \mu=\sum_{i \geqslant s}[m]_{i}$. Define a binary operation on $\mathbb{N}$ by $l \# m=\lambda+\mu+2^{s}-1$. Observe that $l, m \leqslant l \# m \leqslant l+m$, with $l \# m=l+m$ if, and only if $s=0$, that is, $l \perp m$.

Example 7. The binary expansions of 146 and 1304 are $146=2+2^{4}+2^{7}$ and $1304=2^{3}+2^{4}+2^{8}+$ $2^{10}$. Thus, $146 \not 11304$, and in this case $s=5$. It follows that $146 \# 1304=2^{10}+2^{8}+2^{7}+\left(2^{5}-1\right)=$ 1439 .

We are now ready to state the main theorem of this article, which gives the Loewy lengths of tensor products of modules with simple top and simple socle. The remaining sections are dedicated to the proof of this theorem.

Theorem 8. Let $l, m \in \mathbb{N}, l_{1}, l_{2}, m_{1}, m_{2} \in \mathbb{N} \backslash\{0\}, \rho, \sigma \in k \backslash\{0\}$.

1. String with string:

$$
\begin{aligned}
& \ell\left(M\left(A_{l}\right) \otimes M\left(B_{m}\right)\right)= \begin{cases}1+l \# m=1+l+m & \text { if } l \perp m, \\
2+l \# m & \text { if } l \not \perp m,\end{cases} \\
& \ell\left(M\left(A_{l}\right) \otimes M\left(A_{m}\right)\right)= \begin{cases}1+l \# m & \text { if }[l]_{t}=[m]_{t}=0 \text { for all } 0 \leqslant t<s-1, \\
2+l \# m & \text { otherwise. }\end{cases}
\end{aligned}
$$

where $s=\min \left\{r \in \mathbb{N} \mid[l]_{t}+[m]_{t} \leqslant 1, \forall t \geqslant r\right\}$.

2. Band with string:

$$
\ell\left(M\left(A_{l_{1}} B_{l_{2}}^{-1}, \rho\right) \otimes M\left(A_{m}\right)\right)= \begin{cases}2+\left(l_{1}-1\right) \# m & \text { if } \rho=1, l_{1}=l_{2} \text { and } \\ \ell\left(M\left(A_{l_{1}} B_{l_{2}}^{-1}\right) \otimes M\left(A_{m}\right)\right) & l_{1} \perp m, l_{1} \perp(m-1), \\ \text { otherwise. }\end{cases}
$$

3. Band with band: Let $M=M\left(A_{l_{1}} B_{l_{2}}^{-1}, \rho\right), N=M\left(A_{m_{1}} B_{m_{2}}^{-1}, \sigma\right)$.

(a) If $l_{1} \neq l_{2}$, then

$$
\ell(M \otimes N)=\ell\left(M\left(A_{l_{1}} B_{l_{2}}^{-1}\right) \otimes N\right) .
$$

Assume $l_{1}=l_{2}, m_{1}=m_{2}$.

(b) If $l_{1} \not \perp m_{1}, l_{1} \not \perp\left(m_{1}-1\right),\left(l_{1}-1\right) \not \perp m_{1}$ then

$$
\ell(M \otimes N)=2+\left(l_{1}-1\right) \#\left(m_{1}-1\right)=2+l_{1} \# m_{1} .
$$

(c) If $l_{1} \perp m_{1},\left(l_{1}-1\right) \perp m_{1}$, then

$$
\ell(M \otimes N)= \begin{cases}2+\left(l_{1}-1\right) \#\left(m_{1}-1\right) & \text { if } \sigma=1, \\ l_{1}+m_{1}+1 & \text { otherwise. }\end{cases}
$$


(d) If $l_{1} \perp m_{1}, l_{1} \perp\left(m_{1}-1\right)$, then

$$
\ell(M \otimes N)= \begin{cases}2+\left(l_{1}-1\right) \#\left(m_{1}-1\right) & \text { if } \rho=1, \\ l_{1}+m_{1}+1 & \text { otherwise. }\end{cases}
$$

(e) If $\left(l_{1}-1\right) \perp m_{1}, l_{1} \perp\left(m_{1}-1\right)$, then

$$
\ell(M \otimes N)= \begin{cases}2+\left(l_{1}-1\right) \#\left(m_{1}-1\right) & \text { if } \rho=\sigma=1, \\ l_{1}+m_{1} & \text { if } \rho=\sigma \neq 1, \\ l_{1}+m_{1}+1 & \text { otherwise. }\end{cases}
$$

We remark that if any of the statements $l \perp m,(l-1) \perp m$ and $l \perp(m-1)$ holds true, then so does precisely one of the remaining two. Hence the cases listed in item 3 above are all possible. Furthermore, in cases 1 and 2 of Theorem 8, the identities obtained by interchanging the letters $A$ and $B$ also hold true. This can be seen by observing that $X \mapsto Y, Y \mapsto X$ defines an automorphism of $k D_{4 q}$, sending $A_{t}$ to $B_{t}$ and vice versa.

We can now answer the question of when the tensor product of two $k D_{4 q}$-modules contains a projective direct summand. By Proposition 5 it suffices to consider modules with simple top and simple socle. Hence we need only to read off from Theorem 8 when the tensor product of two such modules has Loewy length $2 q+1$.

Corollary 9. Let $l, m<2 q, 0<l_{1}, l_{2}, m_{1}, m_{2}<2 q$, and $\rho, \sigma \in k \backslash\{0\}$.

1. $M\left(A_{l}\right) \otimes M\left(B_{m}\right)$ has a projective direct summand if, and only if, $l+m \geqslant 2 q$,

2. $M\left(A_{l}\right) \otimes M\left(A_{m}\right)$ has a projective direct summand if, and only if, $l+m \geqslant 2 q+1$.

3. $M\left(A_{l_{1}} B_{l_{2}}^{-1}, \rho\right) \otimes M\left(A_{m}\right)$ has a projective direct summand precisely when

$$
\max \left\{l_{1}+m-1, l_{2}+m\right\} \geqslant 2 q .
$$

4. If $l_{1} \neq l_{2}$ or $m_{1} \neq m_{2}$, then $M\left(A_{l_{1}} B_{l_{2}}^{-1}, \rho\right) \otimes M\left(A_{m_{1}} B_{m_{2}}^{-1}, \sigma\right)$ has a projective direct summand if, and only if,

$$
\max \left\{l_{1}+m_{1}-1, l_{1}+m_{2}, l_{2}+m_{1}, l_{2}+m_{2}-1\right\} \geqslant 2 q .
$$

5. If $l_{1}=l_{2}, m_{1}=m_{2}$ then $M\left(A_{l_{1}} B_{l_{2}}^{-1}, \rho\right) \otimes M\left(A_{m_{1}} B_{m_{2}}^{-1}, \sigma\right)$ has projective direct summands if, and only if,

(a) $l_{1} \not \perp\left(m_{1}-1\right)$, and $l_{1}+m_{1} \geqslant 2 q$, or

(b) $l_{1} \perp\left(m_{1}-1\right), \rho \neq \sigma$ and $l_{1}+m_{1}=2 q$.

For $l, m<2 q$, the condition $l+m \geqslant 2 q$ implies $l \not \perp m$. Thus, in particular, in 5(a) above, the condition $l_{1} \perp\left(m_{1}-1\right)$ is equivalent to $\left(l_{1}-1\right) \perp m_{1}$, and similarly, in $5(\mathrm{~b}), l_{1} \not \perp\left(m_{1}-1\right)$ could be replaced by $\left(l_{1}-1\right) \not \perp m_{1}$.

Proof. A $k D_{4 q}$-module has a projective summand if, and only if, its Loewy length equals $2 q+1$. Observe that if $M$ and $N$ are $k D_{4 q}$-modules with $\ell(M)=l+1$ and $\ell(N)=m+1$, then $\ell(M \otimes N) \leqslant$ $l+m+1$. In particular, if $l \perp m$, then $\ell(M \otimes N) \leqslant l+m+1 \leqslant 2 q$, so $M \otimes N$ contains no projective summands. From here on we assume $l, m<2 q$ and $l \not \perp m$.

From the definition it is clear that $l \# m \leqslant 2 q-1$. Moreover, $l \# m=2 q-1$ if, and only if, $[l]_{r}+[m]_{r}=1$ for all $r \in\left\{s, s+1, \ldots, \log _{2}(q)\right\}$ (here $s \in \mathbb{N}$ is as in the definition of $l \# m$ ), which is equivalent to $l+m \geqslant 2 q$. Now, it follows from Theorem 8:1 that $\ell\left(M\left(A_{l}\right) \otimes M\left(B_{m}\right)\right)=2 q+1$ precisely when $l+m \geqslant 2 q$. As for $M\left(A_{l}\right) \otimes M\left(A_{m}\right)$, its Loewy length is $2 q+1$ if, and only if, $l+m \geqslant 2 q$ and there exists a $t<s-1$ such that $[l]_{t}=1$ or $[m]_{t}=1$. This is equivalent to $l+m \geqslant 2 q+1$.

For 3 , note that, by Proposition 5 and $1-2$ above, $\max \left\{l_{1}+m-1, l_{2}+m\right\} \geqslant 2 q$ if, and only if, $M\left(A_{l_{1}} B_{l_{2}}^{-1}\right) \otimes M\left(A_{m}\right)$ has a projective direct summand. Now Theorem 8:2 tells us that $\ell\left(M\left(A_{l_{1}} B_{l_{2}}^{-1}, \rho\right) \otimes M\left(A_{m}\right)\right)=\ell\left(M\left(A_{l_{1}} B_{l_{2}}^{-1}\right) \otimes M\left(A_{m}\right)\right)$, unless $l_{1}=l_{2}, l_{1} \perp m, l_{1} \perp(m-1)$ and $\rho=1$. In the latter case, $\ell\left(M\left(A_{l_{1}} B_{l_{2}}^{-1}\right) \otimes M\left(A_{m}\right)\right) \leqslant l_{1}+m=\max \left\{l_{1}+m-1, l_{2}+m\right\}<2 q$ and no projective summands appear. This proves the result in this case. 
Let $M=M\left(A_{l_{1}} B_{l_{2}}^{-1}, \rho\right)$ and $N=M\left(A_{m_{1}} B_{m_{2}}^{-1}, \sigma\right)$. If $l_{1} \neq l_{2}$ then $\ell(M \otimes N)=\ell\left(M\left(A_{l_{1}} B_{l_{2}}^{-1}\right) \otimes\right.$ $N)$ by Theorem 8:3(a), whence the result follows from 1-3 above. The case $m_{1} \neq m_{2}$, of course, is analogous.

It remains to prove 5. Assume $l_{1}=l_{2}$ and $m_{1}=m_{2}$. Clearly, if $l_{1} \perp m_{1}$ then $l_{1}+m_{1}<2 q$ and $M \otimes N$ has no projective summand. Suppose instead that $l_{1} \not \perp m_{1}$. If $l_{1} \not \perp\left(m_{1}-1\right)$ then also $\left(l_{1}-1\right) \not \perp m_{1}$, and Theorem 8:3(b) means that $M \otimes N$ has a projective summand if, and only if, $2+l_{1} \# m_{1}=2 q+1$, that is, if and only if $l_{1}+m_{1} \geqslant 2 q$. If $l_{1} \perp\left(m_{1}-1\right)$ then also $\left(l_{1}-1\right) \perp m_{1}$, and we are in the situation of Theorem 8:3(e). The condition $\left(l_{1}-1\right) \perp m_{1}$ means that $2+\left(l_{1}-1\right) \#\left(m_{1}-1\right) \leqslant l_{1}+m_{1} \leqslant 2 q$, consequently, $\ell(M \otimes N)<2 q+1$ whenever $\rho=\sigma$. If $\rho \neq \sigma$ then $\ell(M \otimes N)=l_{1}+m_{1}+1$, so $M \otimes N$ has a projective direct summand precisely when $l_{1}+m_{1}=2 q$.

Example 10. In many cases the Loewy length of a tensor products $M \otimes N$ of indecomposable modules $M$ and $N$ can be reduced to determining the maximum of the Loewy lengths of the tensor products of uniserial modules corresponding to the directed components of the defining words for $M$ and $N$, by Proposition 5 and Theorem 8. An example will be illuminating. We consider a tensor product of band modules $M=M\left(A_{l_{1}} B_{l_{2}}^{-1}, \varphi\right)$ and $N=M\left(A_{m_{1}} B_{m_{2}}^{-1}, \psi\right)$, where $\varphi$ and $\psi$ are indecomposable automorphisms of $k^{a}$ and $k^{b}$ respectively.

1. If $a, b>1$ then, by Proposition 5,

$$
\begin{aligned}
\ell(M \otimes N)=\max \left\{\ell\left(M\left(A_{l_{1}}\right) \otimes M\left(A_{m_{1}}\right)\right), \ell\left(M\left(B_{l_{2}}\right) \otimes M\left(A_{m_{1}}\right)\right),\right. \\
\left.\quad \ell\left(M\left(A_{l_{1}}\right) \otimes M\left(B_{m_{2}}\right)\right), \ell\left(M\left(B_{l_{2}}\right) \otimes M\left(B_{m_{2}}\right)\right)\right\} .
\end{aligned}
$$

2. If $a>1, b=1$, then $\ell(M \otimes N)=\max \left\{\ell\left(M\left(A_{l_{1}}\right) \otimes N\right), \ell\left(M\left(B_{l_{2}}\right) \otimes N\right)\right\}$, the value of which is given by Theorem 8:2. Analogously, $\ell(M \otimes N)=\max \left\{\ell\left(M \otimes M\left(A_{m_{1}}\right)\right), \ell\left(M \otimes M\left(B_{m_{2}}\right)\right)\right\}$ if $a=1, b>1$.

Assume that $a=b=1$, so $\varphi, \psi \in k \backslash\{0\}$.

3. If $l_{1} \neq l_{2}$ then from Theorem 8:3(a) and Proposition 5 follows

$$
\ell(M \otimes N)=\max \left\{\ell\left(M\left(A_{l_{1}}\right) \otimes N\right), \ell\left(M\left(B_{l_{2}}\right) \otimes N\right)\right\},
$$

which is computed in Theorem 8:2. In particular, if $\psi \neq 1$, a further application of Proposition 5 reduces this once again to

$$
\begin{aligned}
\ell(M \otimes N)=\max \left\{\ell\left(M\left(A_{l_{1}}\right) \otimes M\left(A_{m_{1}}\right)\right), \ell\left(M\left(B_{l_{2}}\right) \otimes M\left(A_{m_{1}}\right)\right),\right. \\
\left.\quad \ell\left(M\left(A_{l_{1}}\right) \otimes M\left(B_{m_{2}}\right)\right), \ell\left(M\left(B_{l_{2}}\right) \otimes M\left(B_{m_{2}}\right)\right)\right\} .
\end{aligned}
$$

Of course, the case $m_{1} \neq m_{2}$ is analogous.

4. If $l_{1}=l_{2}$ and $m_{1}=m_{2}$, then $\ell(M \otimes N)$ is given directly by Theorem 8:3(b)-(e).

We consider the case $l=146$ and $m=266$, and calculate $\ell\left(M\left(A_{l} B_{l}^{-1}, 1\right) \otimes M\left(A_{m} B_{m}^{-1}, 1\right)\right)$. The binary expansions of $l$ and $m$ are

$$
146=2+2^{4}+2^{7} \text { and } 266=2+2^{3}+2^{8} .
$$

Thus, we have $(l-1) \perp m$ and $l \perp(m-1)$ whilst $l \not \perp m$. Theorem 8:3(e) now gives $\ell(M \otimes N)=$ $2+(l-1) \#(m-1)=411$. In contrast, $l \# m=411$, and hence by Theorem 8:1, we have

$$
\ell\left(M\left(A_{146}\right) \otimes M\left(A_{266}\right)\right)=412 \text { and } \ell\left(M\left(A_{146}\right) \otimes M\left(B_{266}\right)\right)=413 .
$$

In fact, the difference between $\ell(M \otimes N)$ and the maximum of Loewy lengths of tensor products of uniserial modules given by the directed components of the words defining $M$ and $N$ can be arbitrarily large, as demonstrated in the following example.

Example 11. Let $l, m \in \mathbb{N}$ be such that $(l-1) \perp m$ and $l \perp(m-1)$, that is, $l=\lambda+2^{a}, m=\mu+2^{a}$ with $a \in \mathbb{N}, \lambda \perp \mu$ and $2^{a+1} \mid \lambda, \mu$. In particular, $\nu(l)=\nu(m)=a$. Then, by Theorem 8:3(e),

$$
\ell\left(M\left(A_{l} B_{l}^{-1}, 1\right) \otimes M\left(A_{m} B_{m}^{-1}, 1\right)\right)=2+(l-1) \#(m-1)=2+\lambda+\mu+\left(2^{a}-1\right),
$$


whilst

$$
\begin{aligned}
& \max \left\{\ell\left(M\left(A_{l}\right) \otimes M\left(A_{m}\right)\right), \ell\left(M\left(B_{l}\right) \otimes M\left(A_{m}\right)\right), \ell\left(M\left(A_{l}\right) \otimes M\left(B_{m}\right)\right), \ell\left(M\left(B_{l}\right) \otimes M\left(B_{m}\right)\right)\right\} \\
& \quad=\ell\left(M\left(A_{l}\right) \otimes M\left(B_{m}\right)\right)=2+l \# m=2+\lambda+\mu+\left(2^{a+1}-1\right) \\
& \quad=\ell\left(M\left(A_{l} B_{l}^{-1}, 1\right) \otimes M\left(A_{m} B_{m}^{-1}, 1\right)\right)+2^{a} .
\end{aligned}
$$

\section{BASIC SETUP}

In view of the isomorphism (1), we may consider any $M \in \bmod k D_{4 q}$ as a module of the algebra $k\langle X, Y\rangle /\left(X^{2}, Y^{2},(X Y)^{q}+(Y X)^{q}\right)$. The module structure of the tensor product $M \otimes N$ of two modules $M, N \in \bmod k D_{4 q}$ is given by

$$
\begin{aligned}
& X(m \otimes n)=X m \otimes n+m \otimes X n+X m \otimes X n, \\
& Y(m \otimes n)=Y m \otimes n+m \otimes Y n+Y m \otimes Y n
\end{aligned}
$$

for $m \in M$ and $n \in N$. We analyse this action in terms of a quiver representation, as is described in the remainder of this section. Define a quiver $\Gamma$ as follows:

$$
\begin{aligned}
& \Gamma_{0}=\mathbb{N} \times \mathbb{N}, \quad \Gamma_{1}=\left\{\alpha_{i, j}, \beta_{i, j}, \gamma_{l, m} \mid(i, j),(l, m) \in \mathbb{N} \times \mathbb{N}, l+m \in 2 \mathbb{N}\right\}, \\
& \qquad\left\{\begin{array}{l}
\alpha_{i, j}:(i, j) \rightarrow(i, j+1), \\
\beta_{i, j}:(i, j) \rightarrow(i+1, j), \\
\gamma_{i, j}:(i, j) \rightarrow(i+1, j+1) .
\end{array}\right.
\end{aligned}
$$

Let $V$ be the characteristic representation of $\Gamma$, that is, the representation obtained by setting $V_{(i, j)}=k$ for all vertices $(i, j) \in \Gamma_{0}$, and $V_{a}=\mathbb{I}_{k}$ for all arrows $a \in \Gamma_{1}$. We write $1_{(i, j)}$, or simply $(i, j)$ when the context is clear, for the identity element of $k=V_{(i, j)}$ at the vertex $(i, j) \in \Gamma_{0}$. For $r, s \in \mathbb{N}$, let $V(r, s)=V / I(r, s)$ where $I(r, s)=\operatorname{span}_{k}\left\{1_{(i, j)} \mid i>r\right.$ or $\left.j>s\right\} \subset V$ is the subrepresentation generated by all elements $1_{(r+1, j)}$ and $1_{(i, s+1)}, i, j \in \mathbb{N}$.

Set

$$
X \cdot(i, j)= \begin{cases}0 & \text { if } i, j \equiv 0, \\ (i+1, j) & \text { if } i \equiv 1, j \equiv 0, \\ (i, j+1) & \text { if } i \equiv 0, j \equiv 1, \\ (i+1, j)+(i, j+1)+(i+1, j+1) & \text { if } i, j \equiv 1,\end{cases}
$$

and

$$
Y \cdot(i, j)= \begin{cases}0 & \text { if } i, j \equiv 1, \\ (i+1, j) & \text { if } i \equiv 0, j \equiv 1, \\ (i, j+1) & \text { if } i \equiv 1, j \equiv 0, \\ (i+1, j)+(i, j+1)+(i+1, j+1) & \text { if } i, j \equiv 0\end{cases}
$$

This gives $V$ the structure of an (infinite-dimensional) $\Lambda_{0}$-module. Since $I(r, s)$ is closed under the $\Lambda_{0}$-action, $V(r, s)$ also carries a $\Lambda_{0}$-module structure induced from $V$.

Let $M=M\left(A_{m}\right)$ and $N=M\left(A_{n}\right)$. The two modules have standard bases $\left\{A_{r}(u)\right\}_{r \in\{0, \ldots, m\}}$ and $\left\{A_{s}(v)\right\}_{s \in\{0, \ldots, n\}}$ respectively, where $u \in M$ and $v \in N$ are top basis elements. Define linear $\operatorname{maps} \varphi: M \otimes N \rightarrow V(m, n)$ by $\varphi\left(A_{r}(u) \otimes A_{s}(v)\right)=(r, s)$ and $\omega: V \rightarrow V$ by

$$
\omega(i, j)=\sum_{a \in \Gamma_{1}} a(i, j)
$$

Proposition 12. 1. The map $\varphi$ is an isomorphism of $\Lambda_{0}$-modules.

2. The map $\omega$ is injective.

3. Let $r, s, t \in \mathbb{N}$. Then

$$
\begin{array}{lll}
A_{t} \cdot(r, s)=\omega^{t}(r, s), & B_{t} \cdot((r, s+1)+(r+1, s))=\omega^{t}(r+1, s+1) & \text { if } \quad r \equiv s \equiv 0, \\
B_{t} \cdot(r, s)=\omega^{t}(r, s), & A_{t} \cdot((r, s+1)+(r+1, s))=\omega^{t}(r+1, s+1) & \text { if } \quad r \equiv s \equiv 1 .
\end{array}
$$


Proof. The proof of 1 is an easy verification.

For 2, consider a point $x=\sum_{i, j} \lambda_{i, j}(i, j)$ (where $\lambda_{i, j} \in k$ ) in the kernel of $\omega$. Suppose that there exists a point $(l, m) \in \Gamma_{0}$ such that $\lambda_{l, m} \neq 0$, and assume that the natural number $m$ is minimal with this property. We have either $\omega(l, m)=(l+1, m)+(l, m+1)$ or $\omega(l, m)=(l+1, m)+(l, m+1)+$ $(l+1, m+1)$, in both cases, $\omega\left(\lambda_{l, m}(l, m)\right)$ has a homogeneous component $\lambda_{l, m}(l+1, m) \neq 0$. Now $\omega\left(x-\lambda_{l, m}(l, m)\right)=-\lambda_{l, m} \omega(l, m) \neq 0$ hence, since $\omega(i, j) \in \operatorname{span}\{(i+1, j),(i, j+1),(i+1, j+1)\}$, it follows that either $\lambda_{l, m-1}$ or $\lambda_{l-1, m-1}$ is non-zero, contradicting the minimality of $m$. Hence $x=0$, which proves that $\omega$ is injective.

To prove 3 , we suppose that $r \equiv s$ and compute

$$
\begin{aligned}
& \omega(r, s)=(r, s+1)+(r+1, s)+(r+1, s+1), \\
& \omega((r, s+1)+(r+1, s))=(r, s+2)+(r+2, s)
\end{aligned}
$$

hence

$$
\begin{array}{llll}
A_{1} \cdot(r, s)=Y \cdot(r, s)=\omega(r, s), & B_{1} \cdot((l, m+1)+(l+1, m))=\omega(l+1, m+1) & \text { if } \quad r, s \equiv 0, \\
B_{1} \cdot(r, s)=X \cdot(r, s)=\omega(r, s), & A_{1} \cdot((l, m+1)+(l+1, m))=\omega(l+1, m+1) & \text { if } \quad r, s \equiv 1 .
\end{array}
$$

Now let $r, s \equiv 0, u \geqslant 1$ and assume, by induction, that 3 holds for all $t<u$. Then

$$
\begin{aligned}
B_{u}(r, s) & =A_{u-1} Y(r, s)=A_{u-1}((r, s+1)+(r+1, s)+(r+1, s+1)) \\
& =\omega^{u-1}((r, s+1)+(r+1, s))+\omega^{u-1}(r+1, s+1)=\omega^{u}(r, s) .
\end{aligned}
$$

The other identities are proved similarly.

Lemma 13.

$$
\omega^{t}(0,0)=\sum_{l, m \in \mathbb{N}} Q_{t}^{(l, m)}(l, m)
$$

where

$$
Q_{t}^{(l, m)}=\left(\begin{array}{c}
2 t-l-m \\
t-m
\end{array}\right)\left(\begin{array}{c}
\left\lfloor\frac{l+m}{2}\right\rfloor \\
l+m-t
\end{array}\right)=\left(\begin{array}{c}
2 t-l-m \\
t-l
\end{array}\right)\left(\begin{array}{c}
\left\lfloor\frac{l+m}{2}\right\rfloor \\
l+m-t
\end{array}\right)
$$

is the number of paths in $\Gamma$ from $(0,0)$ to $(l, m)$ of length $t$.

Proof. Defining $Q_{t}^{(l, m)}$ as the number of paths of length $t$ from $(0,0)$ to $(l, m)$, the expression for $\omega^{t}(0,0)$ certainly holds.

Let $P_{j}^{(l, m)}$ be the number of paths from $(0,0)$ to $(l, m)$ containing precisely $j$ diagonal arrows $\gamma_{r, s}$. Then $Q_{t}^{(l, m)}=P_{l+m-t}^{(l, m)}$.

To find an expression for $P_{j}^{(l, m)}$, consider a path from $(0,0)$ to $(l, m)$ with $j$ arrows of type $\gamma$, it then has $l-j$ arrows of type $\alpha$ and $m-j$ arrows of type $\beta$. For every point $(r, s)$ in $\Gamma$, there is one arrow of type $\alpha$ and one arrow of type $\beta$ starting in $(r, s)$ (namely, $\alpha_{r, s}$ respectively $\beta_{r, s}$ ), hence there are $\left(\begin{array}{c}l+m-2 j \\ l-j\end{array}\right)$ ways of choosing the mutual order of all arrows of type $\alpha$ respectively $\beta$.

Next choose where in the string of arrows $\alpha$ and $\beta$ that the arrows $\gamma$ are to be inserted. A $\gamma$ could be inserted at points preceded by an even number of arrows $\alpha$ and $\beta$, and multiple arrows $\gamma$ could be inserted at each point. There are $\left\lfloor\frac{l+m}{2}\right\rfloor$ such points, so the number of choices is $\left.\left(\frac{\lfloor+m}{2}\right\rfloor\right)$. This proves that $P_{j}^{(l, m)}=\left(\begin{array}{c}l+m-2 j \\ l-j\end{array}\right)\left(\begin{array}{c}\left\lfloor\frac{l+m}{2}\right\rfloor \\ j\end{array}\right\rfloor$, and hence $Q_{t}^{(l, m)}=P_{l+m-t}^{(l, m)}=\left(\begin{array}{c}2 t-l-m \\ t-m\end{array}\right)\left(\begin{array}{c}\left\lfloor\frac{l+m}{2}\right\rfloor \\ l+m-t\end{array}\right)$.

The following properties of $Q_{t}^{(l, m)}$ are easily derived from the definition: 


$$
\begin{aligned}
Q_{t}^{(l, m)} & =Q_{t}^{(m, l)}, \\
Q_{t}^{(l, m)} & \neq 0 \quad \text { only if } \quad \max \{l, m\} \leqslant t \leqslant l+m, \\
Q_{t}^{(l, l)} & =\left(\begin{array}{c}
2(t-l) \\
t-l
\end{array}\right)\left(\begin{array}{c}
l \\
2 l-t
\end{array}\right)=\delta_{t, l}, \\
Q_{t}^{(l, 0)} & =\left(\begin{array}{c}
2 t-l \\
t
\end{array}\right)\left(\begin{array}{c}
\left\lfloor\frac{l}{2}\right\rfloor \\
l-t
\end{array}\right)=\delta_{t, l}, \\
Q_{l+m}^{(l, m)} & =\left(\begin{array}{c}
l+m \\
l
\end{array}\right) .
\end{aligned}
$$

\section{BACK DIAGONALITY}

The following proposition plays a key role in the determination of the Loewy length of tensor products of uniserial modules. While Proposition 1 tells us that the length of a module generated by a homogeneous basis element is expressible in terms of the elements $\omega^{t}(0,0)=\sum_{l, m \in \mathbb{N}} Q_{t}^{(l, m)}(l, m)$, $t \in \mathbb{N}$, the result below basically means that all terms in this sum except the ones for which $l+m=t$ can be disregarded.

Proposition 14. Let $t \in \mathbb{N}$ and suppose that $Q_{t}^{(l, m)} \equiv 1$. Then there exists $l^{\prime} \leqslant l$, and $m^{\prime} \leqslant m$ such that $l^{\prime}+m^{\prime}=t$ and $Q_{t}^{\left(l^{\prime}, m^{\prime}\right)} \equiv 1$.

The remainder of this subsection is dedicated to the proof of Proposition 14. The setup is the following: Assume that $Q_{t}^{(l, m)} \equiv 1$ and $l+m-t=j>0$ (so that $P_{j}^{(l, m)} \equiv 1$ ). Then we need to show that there exist $l^{\prime} \leqslant l$ and $m^{\prime} \leqslant m$ such that $Q_{t}^{\left(l^{\prime}, m^{\prime}\right)} \equiv 1$ and $j^{\prime}=l^{\prime}+m^{\prime}-t<j$, whence the statement of Proposition 14 follows by induction. Observe that if $l+m=t+j$ then

$$
Q_{t}^{(l, m)}=\left(\begin{array}{c}
t-j \\
l-j
\end{array}\right)\left(\begin{array}{c}
\left\lfloor\frac{t+j}{2}\right\rfloor \\
j
\end{array}\right) \equiv\left(\begin{array}{c}
t-j \\
l-j
\end{array}\right)\left(\begin{array}{c}
t+j \\
2 j
\end{array}\right)=\left(\begin{array}{c}
t+j \\
l+j
\end{array}\right)\left(\begin{array}{c}
l+j \\
2 j
\end{array}\right)
$$

hence $Q_{t}^{(l, m)} \equiv 1$ if and only if $[t+j]_{i} \geqslant[l+j]_{i} \geqslant[2 j]_{i}$ for all $i \in \mathbb{N}$.

Let $s=\max \left\{\sigma \in \mathbb{N} \mid[j]_{\sigma}=1\right\}$, i.e., $s$ is the highest number such that $j \geqslant 2^{s}$.

Lemma 15. If $2^{s} \nmid t$ then there exist $l^{\prime} \leqslant l$ and $m^{\prime} \leqslant m$ such that $l^{\prime}+m^{\prime}=t$ and $Q_{t}^{\left(l^{\prime}, m^{\prime}\right)} \equiv 1$.

Proof. Since $Q_{t}^{(l, m)} \equiv 1$, we have $Q_{t-1}^{(l, m-1)} \equiv 1$ or $Q_{t-1}^{(l-1, m)} \equiv 1$ or $Q_{t-1}^{(l, m-2)} \equiv Q_{t-1}^{(l-1, m-1)} \equiv$ $Q_{t-1}^{(l-2, m)} \equiv 1$. By induction, we may assume that there exists $\lambda_{0} \leqslant l$ and $\mu_{0} \leqslant m$ such that $\lambda_{0}+\mu_{0}=t-1$ and $Q_{t-1}^{\left(\lambda_{0}, \mu_{0}\right)} \equiv 1$. Applying $\omega$, we see that either $Q_{t-1}^{(\lambda, \mu)} \equiv 1$ for all $\lambda \leqslant l$, $\mu \leqslant m$ such that $\lambda+\mu=t-1$, or otherwise there exist $l^{\prime} \leqslant l, m^{\prime} \leqslant m$ satisfying $l^{\prime}+m^{\prime}=t$ and $Q_{t}^{\left(l^{\prime}, m^{\prime}\right)} \equiv 1$.

Suppose $Q_{t-1}^{(\lambda, \mu)} \equiv 1$ for all $\lambda \leqslant l, \mu \leqslant m$ such that $\lambda+\mu=t-1$. Observe that $Q_{t-1}^{(\lambda, \mu)} \equiv\left(\begin{array}{c}t-1 \\ \lambda\end{array}\right)$ whenever $\lambda+\mu=t-1$, so the above implies that $\left(\begin{array}{c}t-1 \\ l-r\end{array}\right) \equiv 1$ for all $r \in\{0, \ldots, j+1\}$. By Lucas' theorem, $\left(\begin{array}{c}t-1 \\ l-r\end{array}\right) \equiv 1$ is equivalent to $[t-1]_{i} \geqslant[l-r]_{i}$ for all $i \in \mathbb{N}$.

Since $j \geqslant 2^{s}$, we have $2^{s} \mid(l-r+1)$ for some $r \leqslant j$, that is, $[l-r]_{i}=1$ for all $i \in\{0, s-1\}$. Now $[t-1]_{i} \geqslant[l-r]_{i}$ implies that $[t]_{i}=[(t-1)+1]_{i}=0$ for all $i \leqslant s-1$, i.e., $2^{s} \mid t$. The result follows.

From here on, we assume $2^{s} \mid t$.

Lemma 16. 1. $j=2^{s+1}-2^{a}$ for some $a \in\{0, \ldots, s\}$, 2. $2^{s+1} \mid t$ or $j=2^{s}$. 
Proof. 1. The statement amounts to that $j=\sum_{i=a}^{s} 2^{i}$. Since $Q_{t}^{(l, m)} \equiv 1$, we have $\left(\begin{array}{c}t+j \\ 2 j\end{array}\right) \equiv 1$ and, by Lucas' theorem,

$$
\left(\begin{array}{c}
t+j \\
2 j
\end{array}\right) \equiv \prod_{i}\left(\begin{array}{c}
{[t+j]_{i}} \\
{[2 j]_{i}}
\end{array}\right)=\prod_{i=0}^{s-1}\left(\begin{array}{c}
{[j]_{i}} \\
{[j]_{i-1}}
\end{array}\right) \prod_{i \geqslant s}\left(\begin{array}{c}
{[t+j]_{i}} \\
{[j]_{i-1}}
\end{array}\right) .
$$

In particular, this implies $[j]_{i} \geqslant[j]_{i-1}$ for all $i \in\{1, \ldots, s-1\}$. In addition, since $[j]_{s}=1$ by assumption, we get $j=\sum_{i=a}^{s} 2^{i}$ for some $a \leqslant s$.

2. If $j \neq 2^{s}$ then $a<s$, implying that

$$
1=[j]_{s-1}=[2 j]_{s} \leqslant[t+j]_{s}=1-[t]_{s}
$$

that is, $[t]_{s}=0$. Hence $2^{s+1} \mid t$.

Lemma 17. If $2^{s+1} \mid t$ then

$$
\begin{aligned}
& {[t]_{s+1}=[l+j]_{r}=1 \quad \text { for all } r \in\{a+1, \ldots, s+1\}, \quad \text { and }} \\
& {[l+j]_{r}=0 \quad \text { for all } r \in\{0, \ldots, a-1\} \text {. }}
\end{aligned}
$$

Proof. If $2^{s+1} \mid t$ then the binary expansions of $j$ and $t$ are disjoint, so $[t+j]_{i}=[j]_{i}$ if $i \leqslant s$ and $[t+j]_{i}=[t]_{i}$ if $i>s$. The result then follows from the inequality $[t+j]_{i} \geqslant[l+j]_{i} \geqslant[2 j]_{i}$, using Lemma 16:1.

We now have all tools needed to prove Proposition 14. We will proceed in three separate cases, namely:

1. $2^{s+1}\left|t, \quad 2^{a+1}\right| l+j$,

2. $2^{s+1} \mid t, \quad 2^{a+1} \nmid l+j$,

3. $2^{s+1} \nmid t$.

4.1. The case $2^{s+1} \mid t$ and $2^{a+1} \mid l+j$. Set $l^{\prime}=l-2^{a}, j^{\prime}=j-2^{a}$ and $m^{\prime}=t+j^{\prime}-l^{\prime}$. Now

$$
\left(\begin{array}{c}
t+j^{\prime} \\
l^{\prime}+j^{\prime}
\end{array}\right)=\left(\begin{array}{c}
t+j-2^{a} \\
l+j-2^{a+1}
\end{array}\right) \equiv 1, \quad \text { since } \quad\left\{\begin{array}{l}
{[t+j]_{a}=1,[l+j]_{a}=0,} \\
{[l+j]_{a+1}=1,}
\end{array}\right.
$$

and

$$
\left(\begin{array}{c}
l^{\prime}+j^{\prime} \\
2 j^{\prime}
\end{array}\right)=\left(\begin{array}{c}
l+j-2^{a+1} \\
2 j-2^{a+1}
\end{array}\right) \equiv 1, \quad \text { since } \quad[2 j]_{a+1}=[l+j]_{a+1}=1,
$$

and, consequently, $Q_{t}^{\left(l^{\prime}, m^{\prime}\right)} \equiv 1$.

4.2. The case $2^{s+1} \mid t, 2^{a+1} \nmid l+j$. Here taking $l^{\prime}=l, j^{\prime}=j-2^{a}$, we get

$$
\left(\begin{array}{c}
t+j^{\prime} \\
l^{\prime}+j^{\prime}
\end{array}\right)=\left(\begin{array}{c}
t+j-2^{a} \\
l+j-2^{a}
\end{array}\right) \equiv 1, \quad \text { since } \quad[t+j]_{a}=1=[l+j]_{a}
$$

and

$$
\left(\begin{array}{c}
l^{\prime}+j^{\prime} \\
2 j^{\prime}
\end{array}\right)=\left(\begin{array}{c}
l+j-2^{a} \\
2 j-2^{a+1}
\end{array}\right) \equiv 1, \quad \quad \text { since } \quad[2 j]_{a+1}=1 .
$$

Hence $Q_{t}^{\left(l^{\prime}, m^{\prime}\right)} \equiv 1$ for $m^{\prime}=t+j^{\prime}-l^{\prime}$.

4.3. The case $2^{s+1} \nmid t$. Since $2^{s+1} \nmid t$, we have $[t]_{s}=1$ and, by Lemma $16: 2, j=2^{s}$. This implies $[t+j]_{r}=[l+j]_{r}=0$ for all $r \leqslant s$, and $[t+j]_{s+1}=[l+j]_{s+1}=1$. Hence $[t]_{s+1}=[l]_{s+1}=0$, and $[t]_{r}=[t+j]_{r},[l]_{r}=[l+j]_{r}$ for $r>s+1$. It follows that $\left(\begin{array}{l}t \\ l\end{array}\right) \equiv 1$, that is, $Q_{t}^{(l, t-l)} \equiv 1$.

This concludes the proof of Proposition 14. 


\section{Solution to the Uniserial CASE}

Lemma 18. Let $l, m, s, t \in \mathbb{N}$.

1. If $Q_{2^{s}}^{(l, m)} \equiv 1$ then $l=2^{s}$ or $m=2^{s}$.

2. $Q_{2^{s}-1}^{(l, m)} \equiv 1$ for all $(l, m)$ such that $l+m=2^{s}-1$.

3. If $l, m<2^{s} \leqslant t$ then $Q_{t}^{(l, m)}=0$.

Proof. The second statement follows directly form the formula $Q_{l+m}^{(l, m)} \equiv\left(\begin{array}{c}l+m \\ l\end{array}\right)$, since $\left[2^{s}-1\right]_{i}=1$ for all $i \leqslant s-1$.

If $l+m=2^{s}$ then $Q_{2^{s}}^{(l, m)} \equiv 0$ unless $l \in\left\{0,2^{s}\right\}$, that is, unless either $l$ or $m$ equals $2^{s}$. Proposition 14 implies that if $l, m \in \mathbb{N}$ are any numbers satisfying $Q_{2^{s}}^{(l, m)} \equiv 1$, then there exist $l^{\prime} \leqslant l$ and $m^{\prime} \leqslant m$ such that $l^{\prime}+m^{\prime}=2^{s}$ and $Q_{2^{s}}^{\left(l^{\prime}, m^{\prime}\right)} \equiv 1$. So either $l^{\prime}=2^{s}$ or $m^{\prime}=2^{s}$, giving $l \geqslant 2^{s}$ or $m \geqslant 2^{s}$ respectively. On the other hand, by $6, Q_{2^{s}}^{(l, m)}=0$ if either of $l$ and $m$ is greater than $2^{s}$, thus $2^{s} \in\{l, m\}$.

For the third statement, recall that $Q_{t}^{(l, m)}$ is the number of paths in $\Gamma$ from $(0,0)$ to $(l, m)$ of length $t$. From this description follows that, for every $r \leqslant t$,

$$
Q_{t}^{(l, m)}=\sum_{\lambda, \mu} Q_{r}^{(\lambda, \mu)} \cdot \tilde{Q}_{t-r}^{(\lambda, \mu),(l, m)}
$$

where $\tilde{Q}_{t-r}^{(\lambda, \mu),(l, m)}$ denotes the number of paths in $\Gamma$ from $(\lambda, \mu)$ to $(l, m)$ of length $t-r$. Clearly $\tilde{Q}_{t-r}^{(\lambda, \mu),(l, m)}=0$ if either $\lambda>l$ or $\mu>m$. Setting $r=2^{s}$ we have, by the first statement, that $Q_{2^{s}}^{(\lambda, \mu)} \equiv 0$ unless $\lambda=2^{s}$ or $\mu=2^{s}$, but in this case $\tilde{Q}_{t-2^{s}}^{(\lambda, \mu),(l, m)}=0$. Consequently, $Q_{t}^{(l, m)} \equiv 0$.

The key to determining the Loewy lengths in the uniserial case lies in the following proposition, which provides an easy method to compute $\ell(u \otimes v)$ for homogeneous basis elements $u \otimes v$.

Proposition 19. For all $l, m \in \mathbb{N}$ the identity $\max \left\{t \in \mathbb{N} \mid \omega^{t}(0,0) \notin I(l, m)\right\}=l \# m$ holds.

Proof. Set $\tau(l, m)=\max \left\{t \in \mathbb{N} \mid \omega^{t}(0,0) \notin I(l, m)\right\}$. First note that, by Lemma 18:3, if $l, m<2^{a}$ then $\tau(l, m)<2^{a}$. Moreover, Lemma 18:2 means that if $l+m \geqslant 2^{a}-1$ then $\tau(l, m) \geqslant 2^{a}-1$. Hence, $\tau(l, m)=2^{a}-1=l \# m$ whenever $2^{a-1} \leqslant l, m<2^{a}$.

Let $r, s, t \in \mathbb{N}$. From Proposition 14 follows that $\tau(r, s) \geqslant t$ if, and only if, there exist $\rho \leqslant r$ and $\sigma \leqslant s$ with $\rho+\sigma=t$ such that $Q_{t}^{(\rho, \sigma)} \equiv 1$. Now suppose that $r, s \leqslant 2^{a}$, and consider $\rho \leqslant r$ and $\sigma \leqslant s$. If $\rho+\sigma<2^{a}$ then

$$
Q_{\rho+\sigma}^{(\rho, \sigma)} \equiv\left(\begin{array}{c}
\rho+\sigma \\
\rho
\end{array}\right)=\left(\begin{array}{c}
2^{a} \\
2^{a}
\end{array}\right)\left(\begin{array}{c}
\rho+\sigma \\
\rho
\end{array}\right) \equiv\left(\begin{array}{c}
\rho+\sigma+2^{a} \\
\rho+2^{a}
\end{array}\right)=Q_{\rho+2^{a}}^{\left(\rho+2^{a}, \sigma\right)} .
$$

If instead $\rho+\sigma \geqslant 2^{a}$ then $Q_{\rho+\sigma}^{(\rho, \sigma)} \equiv 0 \equiv Q_{\rho+2^{a}+\sigma}^{\left(\rho+2^{a}, \sigma\right)}$ by Lemma 18:1. In each case $Q_{\rho+\sigma}^{(\rho, \sigma)} \equiv Q_{\rho+2^{a}+\sigma}^{\left(\rho+2^{a}, \sigma\right)}$, which proves that $\tau\left(r+2^{a}, s\right)=\tau(r, s)+2^{a}$ for all $r, s<2^{a}$. Taking $l=r+2^{a}$ and $m=s$ we get $\tau(l, m)=2^{a}+\tau\left(l-2^{a}, m\right)$ for all $l, m \in \mathbb{N}$ such that $m<2^{a} \leqslant l<2^{a+1}$. On the other hand, from the definition follows that $l \# m=2^{a}+\left(l-2^{a}\right) \# m$ in this case; now $\tau(l, m)=l \# m$ follows by induction.

We record the following facts about the operation \#. The third statement is an immediate consequence of Proposition 19, the others follow from the definition. Remember that $\nu(x)=$ $\min \left\{j \in \mathbb{N} \mid[x]_{j} \neq 0\right\}$.

Lemma 20. Let $l, m \in \mathbb{N}$.

1. $\max \{l, m\} \leqslant l \# m \leqslant l+m$.

2. $l \# m=l+m \Leftrightarrow l \perp m$.

3. $\lambda \# \mu \leqslant l \# m$ whenever $\lambda \leqslant l$ and $\mu \leqslant m$.

4. If $l \not \perp m$ then $l \# m=(l-1) \# m=l \#(m-1)$.

5. If $l \perp m$ and $\nu(l)<\nu(m)$ then $l \#(m-1)<(l-1) \# m=l \# m-1=l+m-1$.

Formulae for the length of a tensor product of uniserial string modules can now be derived from Proposition 19. 
Proposition 21. Let $l, m \in \mathbb{N}$, and let $s \in \mathbb{N}$ be the smallest number such that $[l]_{r}+[m]_{r} \leqslant 1$ for all $r \geqslant s$. Then

$$
\ell\left(M\left(A_{l}\right) \otimes M\left(B_{m}\right)\right)= \begin{cases}2+l \# m & \text { if } l \not \perp m, \\ 1+l \# m=1+l+m & \text { if } l \perp m,\end{cases}
$$

and

$$
\ell\left(M\left(A_{l}\right) \otimes M\left(A_{m}\right)\right)= \begin{cases}2+l \# m & \text { if there exists } t<s-1 \text { such that }[l]_{t}=1 \text { or }[m]_{t}=1, \\ 1+l \# m & \text { if }[l]_{t}=[m]_{t}=0 \text { for all } t<s-1,\end{cases}
$$

Observe that $\ell\left(M\left(A_{l}\right) \otimes M\left(A_{m}\right)\right) \leqslant \ell\left(M\left(A_{l}\right) \otimes M\left(B_{m}\right)\right)=\min \{2+l \# m, 1+l+m\}$ for all $l, m \in \mathbb{N}$.

Proof. Suppose that $M$ and $N$ are uniserial string modules, with standard bases $\mathcal{B}_{M}$ and $\mathcal{B}_{N}$ respectively. Let $u \in \mathcal{B}_{M}$ and $v \in \mathcal{B}_{N}$ be top basis elements, and set $l=h(u), m=h(v)$. Denote by $u^{\prime}$ the element in $\mathcal{B}_{M}$ satisfying $h\left(u^{\prime}\right)=l-1$ (i.e., $u^{\prime}=X u$ or $u^{\prime}=Y u$, whichever is non-zero), and by $v^{\prime}$ the element in $\mathcal{B}_{N}$ for which $h\left(v^{\prime}\right)=m-1$. By Lemma $3, h(M \otimes N)=\max \{h(a \otimes b) \mid$ $\left.a \in \mathcal{B}_{M}, b \in \mathcal{B}_{N}\right\}$.

Now, if $a \otimes b \in \mathcal{B}_{M} \otimes \mathcal{B}_{N}$ is pure (that is, if both $a$ and $b$ are annihilated by either $X$ or $Y$ ), then $h(a \otimes b)=h(a) \# h(b)$. If on the other hand $X a=0 \neq X b$, then

$h(a \otimes b)=\max \{1+h(Y a \otimes b), 1+h(a \otimes X b)\}=\max \{1+(h(a)-1) \# h(b), 1+h(a) \#(h(b)-1)\}$.

From Lemma 20:3 follows that $\max \left\{h(a \otimes b) \mid a \in \mathcal{B}_{M}, b \in \mathcal{B}_{N}\right\}=\max \left\{h(u \otimes v), h\left(u^{\prime} \otimes v\right), h(u \otimes\right.$ $\left.\left.v^{\prime}\right)\right\}$. Therefore, if $u \otimes v$ is pure then

$$
h(M \otimes N)=\max \{l \# m, 1+(l-2) \# m, 1+(l-1) \#(m-1), 1+l \#(m-2)\},
$$

whereas

$$
\begin{aligned}
h(M \otimes N) & =\max \{1+(l-1) \# m, 1+l \#(m-1),(l-1) \# m, l \#(m-1)\} \\
& =1+\max \{(l-1) \# m, l \#(m-1)\}
\end{aligned}
$$

if $u \otimes v$ is impure.

Starting with the impure case, assume that $M=M\left(A_{l}\right)$ and $N=M\left(B_{m}\right)$. If $l \perp m$ then $\max \{(l-1) \# m, l \#(m-1)\}=l+m-1$, by Lemma $20: 2$ and 5 , hence $h(M \otimes N)=l+m=l \# m$. If $l \not \perp m$ then $(l-1) \# m=l \#(m-1)=l \# m($ Lemma 20:4), so $h(M \otimes N)=1+l \# m$.

It remains to consider the pure case. Let $M=M\left(A_{l}\right), N=M\left(A_{m}\right)$, and let $s \in \mathbb{N}$ be the smallest number such that $[l]_{r}+[m]_{r} \leqslant 1$ for all $r \geqslant s$, as stated in the proposition. If $s=0$ then $l \# m=l+m$ whereas, by Lemma $20: 1,(l-2) \# m,(l-1) \#(m-1), l \#(m-2) \leqslant l+m-2$. Hence $h(M \otimes N)=l \# m$ in this case.

Assume instead that $s>0$, i.e., $l \not \perp m$. Then $[l]_{s-1}=[m]_{s-1}=1$, so we can write $l=$ $\lambda+2^{s-1}+l_{0}$ and $m=\mu+2^{s-1}+m_{0}$ with

$$
\lambda=\sum_{t \geqslant s}[l]_{t} 2^{t}, \quad l_{0}=\sum_{r<s-1}[l]_{r} 2^{r}, \quad \text { and } \quad \mu=\sum_{t \geqslant s}[m]_{t} 2^{t}, \quad m_{0}=\sum_{r<s-1}[m]_{r} 2^{r} .
$$

First, observe that if $l_{0} \neq 0$ then $(l-1) \not \perp m$, and hence, by Lemma 20:4, $(l-2) \# m=(l-1) \# m=$ $l \# m$. Since $(l-1) \#(m-1), l \#(m-2) \leqslant l \# m$ by Lemma $20: 3$, it follows that $h(M \otimes N)=1+l \# m$. The same is true if $m_{0} \neq 0$.

If $l_{0}=m_{0}=0$ then $[l-1]$ and $[m]$ are disjoint, so $\max \{1+(l-2) \# m, 1+(l-1) \#(m-1), 1+$ $l \#(m-2)\} \leqslant l+m-1=(l-1) \# m \leqslant l \# m$, implying that $h(M \otimes N)=l \# m$.

Summarising, we have

$$
h\left(M\left(A_{l}\right) \otimes M\left(A_{m}\right)\right)= \begin{cases}l \# m & \text { if } s=0 \text { or if } s>0 \text { and } l_{0}=m_{0}=0, \\ 1+l \# m & \text { if } s>0 \text { and either } l_{0} \text { or } m_{0} \text { is non-zero. }\end{cases}
$$




\section{THE NON-UNISERIAL CASE}

In the previous section, we computed the Loewy length of tensor products of uniserial modules. In this section, tensor products involving bands with simple top and simple socle are treated. Proposition 22 gives the Loewy length of the tensor product of such a band with a uniserial string module, while Propositions 23, 24 and 25 cover the case of a product of two band modules.

Proposition 22. Let $N=M\left(A_{m}\right)$ and $M=M\left(A_{l_{1}} B_{l_{2}}^{-1}, \rho\right)$. Now

$$
\ell(M \otimes N)= \begin{cases}2+\left(l_{1}-1\right) \# m & \text { if } \rho=1, l_{1}=l_{2} \text { and } l_{1} \perp m, l_{1} \perp(m-1), \\ \ell\left(M\left(A_{l_{1}} B_{l_{2}}^{-1}\right) \otimes N\right) & \text { otherwise. }\end{cases}
$$

Remember that $l \# m=l+m$ if and only if the binary expansions of $l$ and $m$ are disjoint.

Proof. Let $u_{a}$ and $u_{b}$ be top basis elements in $M\left(A_{l_{1}}\right)$ and $M\left(B_{l_{2}}\right)$ respectively, and set $u^{\prime}=u_{a}+u_{b}$. Then $\left\langle u^{\prime}\right\rangle \simeq M\left(A_{l_{1}} B_{l_{2}}^{-1}\right)$ and $M \simeq\left\langle u^{\prime}\right\rangle / U$, where $U=\left\langle A_{l_{1}} u^{\prime}+\rho B_{l_{2}} u^{\prime}\right\rangle=\left\langle A_{l_{1}} u_{a}+\rho B_{l_{2}} u_{b}\right\rangle$. We view the two latter isomorphisms as identifications, and write $M\left(A_{l_{1}} B_{l_{2}}^{-1}\right)=\left\langle u^{\prime}\right\rangle$ and $M=$ $M\left(A_{l_{1}} B_{l_{2}}^{-1}\right) / U$. Moreover, $v$ denotes a top basis element in $N$. Now $M \otimes N \simeq \frac{M\left(A_{l_{1}} B_{l_{2}}^{-1}\right) \otimes N}{U \otimes N}$, and $\ell(M \otimes N) \leqslant \ell\left(M\left(A_{l_{1}} B_{l_{2}}^{-1}\right) \otimes N\right)$.

Assume that $\ell(M \otimes N)<\ell\left(M\left(A_{l_{1}} B_{l_{2}}^{-1}\right) \otimes N\right)$. Then there exists a natural number $t$ such that $\operatorname{rad}^{t}\left(M\left(A_{l_{1}} B_{l_{2}}^{-1}\right) \otimes N\right)$ is non-zero but contained in $U \otimes N$. Now, for any $t \geqslant 0, B_{t}$. $\left(M\left(A_{l_{1}} B_{l_{2}}^{-1}\right) \otimes N\right) \not \subset U \otimes N$ unless $B_{t} \cdot\left(M\left(A_{l_{1}} B_{l_{2}}^{-1}\right) \otimes N\right)=0$, so the above condition implies $0 \neq A_{t} \cdot\left(M\left(A_{l_{1}} B_{l_{2}}^{-1}\right) \otimes N\right) \subset U \otimes N$ for some $t$. The latter can be true only if $A_{t} \cdot\left(u^{\prime} \otimes v\right) \in$ $(U \otimes N) \backslash\{0\}$, equivalently, there exists a non-zero $n \in N$ such that $A_{t} \cdot\left(u_{a} \otimes v\right)=A_{l_{1}} u_{a} \otimes n$ and $A_{t} \cdot\left(u_{b} \otimes v\right)=\rho B_{l_{2}} u_{b} \otimes n$.

We have $A_{t} \cdot\left(u_{a} \otimes v\right)=\sum_{i, j} \gamma_{i, j} A_{i} u_{a} \otimes A_{j} v$, with $\gamma_{i, j} \in \mathbb{F}_{2}=\{0,1\}$. Hence $A_{t} \cdot\left(u_{a} \otimes v\right)=$ $A_{l_{1}} u_{a} \otimes n$ implies $n=\sum_{j} \gamma_{l_{1}, j} A_{j} v$. On the other hand, $A_{t} \cdot\left(u_{b} \otimes v\right)=\rho B_{l_{2}} u_{b} \otimes n$ similarly gives $n=\sum_{j} \rho \delta_{j} A_{j} v$ for some $\delta_{j} \in\{0,1\}$. Since $n \neq 0$ this means that $\rho=1$.

Next, by Equation (6) and Proposition 14, the identity $A_{t} \cdot\left(u_{a} \otimes v\right)=A_{l_{1}} u_{a} \otimes n$ implies that $\min \left\{j \in \mathbb{N} \mid \delta_{j} \neq 0\right\}=t-l_{1}$, and $A_{t} \cdot\left(u_{b} \otimes v\right)=B_{l_{2}} u_{b} \otimes n \operatorname{implies} \min \left\{j \in \mathbb{N} \mid \delta_{j} \neq 0\right\}=t-l_{2}$. Thus $l_{1}=l_{2}$.

From here on, set $l=l_{1}=l_{2}$. The existence of a non-zero $n \in N$ such that $A_{t} \cdot\left(u_{a} \otimes v\right)=A_{l} u_{a} \otimes n$ and $A_{t} \cdot\left(u_{b} \otimes v\right)=B_{l} u_{b} \otimes n$ is equivalent to $A_{l+m}\left(u_{a} \otimes v\right)$ and $A_{l+m}\left(u_{b} \otimes v\right)$ being non-zero. Namely, as we saw above, $A_{t} \cdot\left(u_{a} \otimes v\right)=A_{l} u_{a} \otimes n$ for $n=\sum_{\mu=0}^{m} \gamma_{\mu} A_{\mu} v \neq 0$ implies that $\min \left\{\mu \mid \gamma_{\mu} \neq 0\right\}=t-l$. Since $A_{l} u_{a} \in \operatorname{soc} M\left(A_{l}\right)$, we have $R \cdot\left(A_{l} u_{a} \otimes n\right)=A_{l} u_{a} \otimes R n$ for all $R \in \operatorname{rad}\left(k D_{4 q}\right)$, and so $A_{t+r} \cdot\left(u_{a} \otimes v\right)=A_{l} u_{a} \otimes\left(\sum_{\mu} \gamma_{\mu} A_{\mu+r} v\right)$ for all $r \in \mathbb{N}$. Setting $r=l+m-t$, this gives

$$
A_{l+m} \cdot\left(u_{a} \otimes v\right)=A_{l} u_{a} \otimes\left(\gamma_{t-l} A_{m} v\right)=A_{l} u_{a} \otimes A_{m} v \neq 0 .
$$

Similarly, one proves that $A_{l+m} \cdot\left(u_{b} \otimes v\right)$ is non-zero if $A_{t} \cdot\left(u_{b} \otimes v\right)=B_{l} u_{b} \otimes n$ for some $t>0$ and $n \in N \backslash\{0\}$.

Now $A_{l+m}\left(u_{a} \otimes v\right) \neq 0$ precisely when $l \# m=l+m$, and $A_{l+m}\left(u_{b} \otimes v\right) \neq 0$ precisely when $l \#(m-1)=l+m-1$. So if either doesn't hold, then $\ell(M \otimes N)=\ell\left(\left\langle u^{\prime}\right\rangle \otimes N\right)=\ell\left(M^{\prime} \otimes N\right)$.

From here on, assume that $A_{l+m}\left(u_{a} \otimes v\right)$ and $A_{l+m}\left(u_{b} \otimes v\right)$ are non-zero. Since the binary expansions of $l$ and $m$ are disjoint, $\nu(l) \neq \nu(m)$, and $l \perp(m-1)$ then implies $\nu(l)>\nu(m)$. Consequently, $(l-1) \not \perp m$, so $(l-1) \# m<l+m-1$ by Lemma 20:2.

We have

$$
h(u \otimes v) \geqslant \max \left\{\tau \mid B_{\tau} \cdot(u \otimes v) \neq 0\right\}=\max \left\{\tau \mid B_{\tau} \cdot\left(M\left(B_{l}\right) \otimes N\right) \neq 0\right\}=1+(l-1) \# m
$$

and it is easy to check that $h(a \otimes b) \leqslant h(u \otimes v)$ for all $a \in \mathcal{B}_{M}, b \in \mathcal{B}_{N}$, hence $h(M \otimes N)=h(u \otimes v)$ by Lemma 3. Set $t=2+(l-1) \# m$. To prove the proposition, it is now enough to show that $A_{t} \cdot(u \otimes v)=0$, since this, together with $(11)$, implies $h(u \otimes v)=1+(l-1) \# m$. 
Observe that

$$
\begin{aligned}
& \min \left\{\tau \in \mathbb{N} \mid A_{\tau} \cdot(u \otimes v) \in(\operatorname{soc} M) \otimes N\right\} \\
= & \min \left\{\tau \in \mathbb{N} \mid A_{\tau} \cdot\left(M\left(A_{l-1}\right) \otimes N\right)=0, A_{\tau} \cdot\left(M\left(B_{l-1}\right) \otimes N\right)=0\right\} \\
= & \max \{1+(l-1) \# m, 2+(l-1) \#(m-1)\} \leqslant t,
\end{aligned}
$$

hence $A_{t} \cdot(u \otimes v) \in(\operatorname{soc} M) \otimes N$. Equivalently, $A_{t} \cdot\left(u_{a} \otimes v\right) \in\left(\operatorname{soc} M\left(A_{l}\right)\right) \otimes N$ and $A_{t} \cdot\left(u_{b} \otimes v\right) \in$ $\left(\operatorname{soc} M\left(B_{l}\right)\right) \otimes N$.

Writing

$$
\begin{aligned}
& A_{\tau} \cdot\left(u_{a} \otimes v\right)=\sum_{\lambda, \mu} \alpha_{\tau}^{\lambda, \mu}\left(A_{\lambda} u_{a}\right) \otimes\left(A_{\mu} v\right) \in M\left(A_{l}\right) \otimes N \quad \text { and } \\
& A_{\tau} \cdot\left(u_{b} \otimes v\right)=\sum_{\lambda, \mu} \beta_{\tau}^{\lambda, \mu}\left(B_{\lambda} u_{b}\right) \otimes\left(A_{\mu} v\right) \in M\left(B_{l}\right) \otimes N
\end{aligned}
$$

we have $\alpha_{\tau}^{\lambda, \mu} \equiv Q_{\tau}^{(\lambda, \mu)}$ and $\beta_{\tau}^{\lambda, \mu} \equiv Q_{\tau-1}^{(\lambda, \mu-1)} \equiv \alpha_{\tau-1}^{\lambda, \mu-1}$. As for $\tau=t$,

$$
\begin{aligned}
& A_{t} \cdot\left(u_{a} \otimes v\right)=\sum_{j=0}^{l+m-t} \alpha_{t}^{l, t+j-l}\left(A_{l} u_{a}\right) \otimes\left(A_{t+j-l} v\right) \quad \text { and } \\
& A_{t} \cdot\left(u_{b} \otimes v\right)=\sum_{j=0}^{l+m-t} \beta_{t}^{l, t+j-l}\left(B_{l} u_{b}\right) \otimes\left(A_{t+j-l} v\right) .
\end{aligned}
$$

Both $A_{t}\left(u_{a} \otimes v\right)$ and $A_{t}\left(u_{b} \otimes v\right)$ are non-zero, since $\min \left\{\tau \mid A_{\tau}\left(u_{a} \otimes v\right)=0\right\}=1+l \# m=$ $1+l+m>l+m \geqslant 2+(l-1) \# m=t$, and similarly $\min \left\{\tau \mid A_{\tau}\left(u_{b} \otimes v\right)=0\right\}=2+l \#(m-1)>t$. Hence, Proposition 14 (together with the fact that $\alpha_{t}^{\lambda, \mu}=\beta_{t}^{\lambda, \mu}=0$ whenever $\lambda \neq l$ ) gives that $\alpha_{t}^{l, t-l}=1$ and $\beta_{t}^{l, t-l}=1$, that is, $\left(\begin{array}{l}t \\ l\end{array}\right) \equiv\left(\begin{array}{c}t-1 \\ l\end{array}\right) \equiv 1$. In particular, $\nu(t)<\nu(l)$.

To conclude the proof of the proposition, we shall show that $\alpha_{t}^{l, t+j-l}=\beta_{t}^{l, t+j-l}$ for all $j \leqslant$ $l+m-t$, which is equivalent to $A_{t}(u \otimes v)=0$. As we have seen, $\alpha_{t}^{l, t-l}=\beta_{t}^{l, t-l}=1$, so consider $j>0$.

First assume that $\alpha_{t}^{l, t+j-l}=1$ and $\nu(j)>\nu(t)$. Since also $\nu(l)>\nu(t)$, it follows that $\nu(l+$ $j) \geqslant \min \{\nu(l), \nu(j)\} \geqslant \nu(t)+1=\nu(t+j)+1$. Hence $2^{\nu(t)+1} \mid(l+j)$ and $t+j=2^{\nu(t)}+T$, where $2^{\nu(t)+1} \mid T$. So $Q_{t}^{(l, t+j-l)} \equiv \alpha_{t}^{l, t+j-l}=1$ implies $\left(\begin{array}{c}T \\ l+j\end{array}\right)=\left(\begin{array}{c}T \\ l+j\end{array}\right)\left(\begin{array}{c}\nu(t) \\ 0\end{array}\right) \equiv\left(\begin{array}{c}t+j \\ l+j\end{array}\right) \equiv 1$. But $t-1+j=T+\left(2^{\nu(t)}-1\right)$, so

$$
\left(\begin{array}{c}
t-1+j \\
l+j
\end{array}\right) \equiv\left(\begin{array}{c}
T \\
l+j
\end{array}\right)\left(\begin{array}{c}
2^{\nu(t)}-1 \\
0
\end{array}\right)=\left(\begin{array}{c}
T \\
l+j
\end{array}\right) \equiv 1,
$$

implying $\beta_{t}^{l, t+j-l} \equiv\left(\begin{array}{c}t-1+j \\ l+j\end{array}\right)\left(\begin{array}{c}l+j \\ 2 j\end{array}\right) \equiv 1$ (clearly, $1=\alpha_{t}^{l, t+j-l} \equiv\left(\begin{array}{c}t+j \\ l+j\end{array}\right)\left(\begin{array}{c}l+j \\ 2 j\end{array}\right)$ means that $\left.\left(\begin{array}{c}l+j \\ 2 j\end{array}\right) \equiv 1\right)$

Next, consider the case $\nu(j) \leqslant \nu(t)$. Here, if $\left(\begin{array}{c}l+j \\ 2 j\end{array}\right) \equiv 1$ then $[j]_{i}=1$ for all $i \in\{\nu(j), \ldots, \nu(l)-1\}$, in particular, $[j]_{\nu(t)}=1$. Hence $[t+j]_{\nu(t)}=0$. But $[l+j]_{\nu(t)}=[j]_{\nu(t)}=1$ since $\nu(l)>\nu(t)$, so $\left(\begin{array}{l}t+j \\ l+j\end{array}\right) \equiv 0$ and $\alpha_{t}^{l, t+j-l}=0$.

The arguments in the two preceding paragraphs show that the inequality $\alpha_{t}^{l, t+j-l} \leqslant \beta_{t}^{l, t+j-l}$ holds true. To prove the converse assume, for a contradiction, that $\beta_{t}^{l, \mu}=1$ and $\alpha_{t}^{l, \mu}=0$, where $\mu=t+j-l$. As $\alpha_{t-1}^{l, \mu-1}=\beta_{t}^{l, \mu}=1$, it follows that either $\alpha_{t-1}^{l-1, \mu-1}=1$ or $\alpha_{t-1}^{l-1, \mu}=1$ (but not both). By Proposition 14, there exist $\lambda_{0} \leqslant l-1$ and $\mu_{0} \leqslant \mu$ satisfying $\lambda_{0}+\mu_{0}=t-1$ such that $\alpha_{t-1}^{\lambda_{0}, \mu_{0}}=1$. As $\alpha_{t}^{r, s}=0$ whenever $r \leqslant l-1$, this means that actually $\alpha_{t-1}^{r, s}=1$ for all $r \leqslant l-1$, $s \leqslant m$ satisfying $r+s=t-1$. In particular, $\alpha_{t-1}^{l-1, t-l}=1$. But $\alpha_{t-1}^{l-1, t-l}=\beta_{t}^{l-1, t-l+1}$, and since $t-l+1=m-j+1 \leqslant m$, this contradicts the assumption that $\beta_{t}^{l-1, s}=0$ for all $s \leqslant m$. This establishes the inequality $\beta_{t}^{l, t+j-l}=1 \leqslant \alpha_{t}^{l, t+j-l}$, concluding the proof of the proposition.

The last step in establishing the proof of Theorem 8 is to determine the Loewy length of a tensor product of two bands with simple top and socle. 
First, we set some notation for the remainder of this section: take $u_{a}$ and $u_{b}$ to be top basis elements in the modules $M\left(A_{l_{1}}\right)$ and $M\left(B_{l_{2}}\right)$ respectively, set $u^{\prime}=u_{a}+u_{b}$ and $M^{\prime}=\left\langle u^{\prime}\right\rangle \subset$ $M\left(A_{l_{1}}\right) \oplus M\left(B_{l_{2}}\right)$. Then $M^{\prime} \simeq M\left(A_{l_{1}} B_{l_{2}}^{-1}\right)$, and setting $M=M^{\prime} / U$, where $U=\left\langle A_{l_{1}} u^{\prime}+\rho B_{l_{2}} u^{\prime}\right\rangle=$ $\left\langle A_{l_{1}} u_{a}+\rho B_{l_{2}} u_{b}\right\rangle \subset M^{\prime}$ and $\rho \in k \backslash\{0\}$, we have $M \simeq M\left(A_{l_{1}} B_{l_{2}}^{-1}, \rho\right)$. Similarly, $v_{a}$ and $v_{b}$ are top basis element of $M\left(A_{m_{1}}\right)$ respectively $M\left(B_{m_{2}}\right), v^{\prime}=v_{a}+v_{b}, N^{\prime}=\left\langle v^{\prime}\right\rangle \subset M\left(A_{m_{1}}\right) \oplus M\left(B_{m_{2}}\right)$, and $N=N^{\prime} / V$ for $V=\left\langle A_{m_{1}} v^{\prime}+\sigma B_{m_{2}} v^{\prime}\right\rangle \subset N^{\prime}, \sigma \in k \backslash\{0\}$. In this way, $N^{\prime} \simeq M\left(A_{m_{1}} B_{m_{2}}^{-1}\right)$ and $N \simeq M\left(A_{m_{1}} B_{m_{2}}^{-1}, \sigma\right)$. We denote by $\mathcal{B}_{M^{\prime}}$ and $\mathcal{B}_{N^{\prime}}$ the standard bases of $M^{\prime}$ and $N^{\prime}$ containing $u^{\prime}$ and $v^{\prime}$ respectively, and by $\mathcal{B}_{M}$ and $\mathcal{B}_{N}$ the corresponding standard bases in $M$ and $N$. The images of $u^{\prime}$ and $v^{\prime}$ under the quotient projections $M^{\prime} \rightarrow M$ and $N^{\prime} \rightarrow N$ are denoted by $u$ and $v$ respectively.

Proposition 23. Assume that $l_{1} \neq l_{2}$ and $m_{1} \neq m_{2}$. Then $\ell(M \otimes N)=\ell\left(M^{\prime} \otimes N^{\prime}\right)$.

Proof. Let $\bar{M}=\operatorname{rad} M \subset M$, and $\underline{M}=M / \operatorname{soc} M$. Set $l=\max \left\{l_{1}, l_{2}\right\}$ and $m=\max \left\{m_{1}, m_{2}\right\}$, and let $x \in\left\{u_{a}, u_{b}\right\}, y \in\left\{v_{a}, v_{b}\right\}$ be the unique elements such that $h(x)=l$ and $h(y)=m$. Clearly,

$$
\max \{h(\bar{M} \otimes N), h(\underline{M} \otimes N)\} \leqslant h(M \otimes N) \leqslant h\left(M^{\prime} \otimes N^{\prime}\right) .
$$

Observe that

$$
\begin{aligned}
\operatorname{rad}^{l+m}\left(M^{\prime} \otimes N^{\prime}\right) & =\operatorname{span}\left\{A_{l+m} \cdot\left(u^{\prime} \otimes v^{\prime}\right), B_{l+m} \cdot\left(u^{\prime} \otimes v^{\prime}\right)\right\} \\
& =\operatorname{span}\left\{A_{l+m} \cdot(x \otimes y), B_{l+m} \cdot(x \otimes y)\right\}
\end{aligned}
$$

which is contained in $U \otimes N^{\prime}+M^{\prime} \otimes V$ only if it is zero. This means that $h(M \otimes N)=l+m$ if, and only if, $h\left(M^{\prime} \otimes N^{\prime}\right)=l+m$, and hence the proposition holds true in case $h\left(M^{\prime} \otimes N^{\prime}\right)=l+m$.

Assume instead that $h\left(M^{\prime} \otimes N^{\prime}\right) \leqslant l+m-1$. Then, in particular, $l \# m \leqslant l+m-1$, that is, $l \not \perp m$. From Proposition 21 follows that $h\left(M^{\prime} \otimes N^{\prime}\right) \leqslant 1+l \# m \leqslant \min \{1+l \# m, l+m-1\}$.

By Proposition 5 and Proposition 22,

$$
\begin{aligned}
& \max \{h(\bar{M} \otimes N), h(\underline{M} \otimes N)\}=\max \left\{M\left(A_{l_{i}-1}\right) \otimes N, M\left(B_{l_{i}-1}\right) \otimes N \mid i=1,2\right\} \\
= & \max \left\{M\left(A_{l-1}\right) \otimes N, M\left(B_{l-1}\right) \otimes N\right\}=\min \{1+(l-1) \# m, l+m-1\} .
\end{aligned}
$$

Since $l \not \perp m$, Lemma 20 gives $(l-1) \# m=l \# m$, hence

$$
\max \{h(\bar{M} \otimes N), h(\underline{M} \otimes N)\}=\min \{1+l \# m, l+m-1\} \geqslant h\left(M^{\prime} \otimes N^{\prime}\right) .
$$

This, together with the inequality (12) shows that $h(M \otimes N)=h\left(M^{\prime} \otimes N\right)=h\left(M^{\prime} \otimes N^{\prime}\right)$ in case $h\left(M^{\prime} \otimes N^{\prime}\right) \leqslant l+m-1$, concluding the proof.

Proposition 24. If $l_{1} \neq l_{2}$ and $m_{1}=m_{2}$, then $\ell(M \otimes N)=\ell\left(M^{\prime} \otimes N\right)$.

Proof. We have $\ell(M \otimes N)<\ell\left(M^{\prime} \otimes N\right)$ if, and only if, there exists a number $t \in \mathbb{N}$ such that $\operatorname{rad}^{t}\left(k D_{4 q}\right) \cdot\left(u^{\prime} \otimes v\right)$ is non-zero but contained in $U \otimes N$. This is equivalent to $A_{t}\left(u^{\prime} \otimes v\right)=$ $\left(A_{l_{1}} u^{\prime}+\rho B_{l_{2}} u^{\prime}\right) \otimes n_{1}$ and $B_{t}\left(u^{\prime} \otimes v\right)=\left(A_{l_{1}} u^{\prime}+\rho B_{l_{2}} u^{\prime}\right) \otimes n_{2}$ with $n_{1}, n_{2} \in N$ not both equal to zero.

Assume that $\ell(M \otimes N)<\ell\left(M^{\prime} \otimes N\right)$, and let $n \in\left\{n_{1}, n_{2}\right\}$ be non-zero. Set $\mu$ to be the largest number such that $n \in \operatorname{rad}^{\mu}(N)$. Then $n=\sum_{j=\mu}^{m_{1}} \gamma_{j} A_{j} v+\sum_{j=\mu}^{m_{1}} \gamma_{j}^{\prime} B_{j} v$, with either $\gamma_{\mu}$ or $\gamma_{\mu}^{\prime}$ non-zero. Now Proposition 14 implies that $l_{1}+\mu=t=l_{2}+\mu$, hence $l_{1}=l_{2}$.

From here on, assume that $l=l_{1}=l_{2}$ and $m=m_{1}=m_{2}$.

\section{Proposition 25.}

1. If $l \not \perp m, l \not \perp(m-1),(l-1) \not \perp m$ then $\ell(M \otimes N)=2+(l-1) \#(m-1)=2+l \# m$.

2. If $l \perp m,(l-1) \perp m$, then $\ell(M \otimes N)= \begin{cases}2+(l-1) \#(m-1) & \text { if } \sigma=1, \\ l+m+1 & \text { otherwise. }\end{cases}$

3. If $l \perp m, l \perp(m-1)$, then $\ell(M \otimes N)= \begin{cases}2+(l-1) \#(m-1) & \text { if } \rho=1, \\ l+m+1 & \text { otherwise. }\end{cases}$ 
4. If $(l-1) \perp m, l \perp(m-1)$, then $\ell(M \otimes N)= \begin{cases}2+(l-1) \#(m-1) & \text { if } \rho=\sigma=1, \\ l+m & \text { if } \rho=\sigma \neq 1, \\ l+m+1 & \text { otherwise. }\end{cases}$

Proof. We shall have reason to use the following formulae:

$$
\begin{aligned}
& A_{l+m} \cdot\left(u^{\prime} \otimes v^{\prime}\right)=\delta_{1} A_{l} u^{\prime} \otimes A_{m} v^{\prime}+\delta_{2} A_{l} u^{\prime} \otimes B_{m} v^{\prime}+\delta_{3} B_{l} u^{\prime} \otimes A_{m} v^{\prime}, \\
& B_{l+m} \cdot\left(u^{\prime} \otimes v^{\prime}\right)=\delta_{1} B_{l} u^{\prime} \otimes B_{m} v^{\prime}+\delta_{2} B_{l} u^{\prime} \otimes A_{m} v^{\prime}+\delta_{3} A_{l} u^{\prime} \otimes B_{m} v^{\prime},
\end{aligned}
$$

where $\delta_{1}=\delta_{l \# m, l+m}, \delta_{2}=\delta_{(l-1) \# m, l+m-1}$ and $\delta_{3}=\delta_{l \#(m-1), l+m-1}$. From this follows that

$$
\begin{aligned}
& A_{l+m} \cdot(u \otimes v)=\left(\sigma \rho \delta_{1}+\rho \delta_{2}+\sigma \delta_{3}\right) B_{l} u \otimes B_{m} v, \\
& B_{l+m} \cdot(u \otimes v)=\left(\delta_{1}+\sigma \delta_{2}+\rho \delta_{3}\right) B_{l} u \otimes B_{m} v,
\end{aligned}
$$

Set $\underline{M}=M / \operatorname{soc} M=M^{\prime} / \operatorname{soc} M^{\prime}$ and $\underline{N}=N / \operatorname{soc} N=N^{\prime} / \operatorname{soc} N^{\prime}$. Observe that by Proposition 5 and Proposition 22,

$$
\begin{aligned}
h(\underline{M} \otimes N) & =\max \left\{h\left(M\left(A_{l-1}\right) \otimes N\right), h\left(M\left(B_{l-1}\right) \otimes N\right)\right\}=h\left(M\left(A_{l-1}\right) \otimes N\right) \\
& = \begin{cases}1+(l-1) \#(m-1) & \text { if } \sigma=1,(l-1) \perp m,(l-2) \perp m, \\
h\left(M\left(A_{l-1}\right) \otimes M\left(B_{m}\right)\right) & \text { otherwise. }\end{cases}
\end{aligned}
$$

Moreover, as $\underline{M}$ is a quotient of $M$, which in turn is a quotient of $M^{\prime}$, we have the following sequence of inequalities:

$1+(l-1) \#(m-1) \leqslant h(\underline{M} \otimes N) \leqslant h(M \otimes N) \leqslant h\left(M^{\prime} \otimes N\right) \leqslant h\left(M^{\prime} \otimes N^{\prime}\right)=\min \{l+m, 1+l \# m\}$, where the last identity follows from Proposition 21. This immediately gives the first statement of the proposition: if $l \not \not m$ and $(l-1) \not \perp m$ (and hence $l \not \not(m-1))$ then $1+(l-1) \#(m-1)=1+l \# m$ by Lemma 20:4, so $h(M \otimes N)=1+(l-1) \#(m-1)=1+l \# m$.

Assume now that $l \perp m,(l-1) \perp m$ (hence $l \not \perp(m-1))$. If $\sigma=1$, then $h\left(M^{\prime} \otimes N\right)=$ $1+l \#(m-1)=1+(l-1) \#(m-1)$ where Proposition 22 gives the first identity and Lemma 20 the second. It follows that $h(M \otimes N)=1+(l-1) \#(m-1)$ in this case. If on the other hand $\sigma \neq 1$, then $A_{l+m}(u \otimes v)=\rho(\sigma+1) B_{l} u \otimes B_{m} v \neq 0$, hence $h(M \otimes N)=l+m$. This proves the second statement of the proposition, whence the third statement follows by symmetry.

For the fourth statement, assume that $(l-1) \perp m$ and $l \perp(m-1)$. Then, from the equations (15) and (16) follows

$$
A_{l+m}(u \otimes v)=B_{l+m}(u \otimes v)=(\rho+\sigma) B_{l} u \otimes B_{m} v
$$

which is zero if, and only if, $\rho=\sigma$. It follows that $h(M \otimes N)=l+m$ if $\rho \neq \sigma$. Assume instead $\rho=\sigma$. Since $h(a \otimes b) \leqslant h(a)+h(b)<l+m$ for any $a \otimes b \in \mathcal{B}_{M} \otimes \mathcal{B}_{N} \backslash\{u \otimes v\}$, we have $h(M \otimes N)<l+m$ in this case. On the other hand, if $\rho \neq 1$ then, by Proposition 22 and Proposition 21,

$$
h(M \otimes N) \geqslant h(M \otimes \underline{N})=h\left(M \otimes M\left(A_{m-1}\right)\right)=h\left(M\left(B_{l}\right) \otimes M\left(A_{m-1}\right)\right)=l+m-1 .
$$

Hence $h(M \otimes N)=l+m-1$ if $\rho=\sigma \neq 1$.

It remains to consider the case $\rho=\sigma=1$. First, suppose that $(l-2) \not \perp m$; then $l+m>$ $h(M \otimes N) \geqslant h(\underline{M} \otimes N)=l+m-1$, so $h(M \otimes N)=l+m-1$. But $(l-2) \not \perp m$ implies that $l=\lambda+1$ and $m=\mu+1$ where $\lambda$ and $\mu$ are even, positive integers satisfying $\lambda \perp \mu$; this means that $1+(l-1) \#(m-1)=1+\lambda+\mu=l+m-1=h(M \otimes N)$.

Next, let $l=1$. Then again $h(\underline{M} \otimes N)=m=l+m-1=1+(l-1) \#(m-1)$, and so $h(M \otimes N)=1+(l-1) \#(m-1)$.

Last, assume that $(l-2) \perp m, l \geqslant 2$. Then $l=\lambda+2^{a}, m=\mu+2^{a}$, where $a \geqslant 1, \lambda \perp \mu$ and $2^{a+1} \mid \lambda, \mu$; hence also $l \perp(m-2)$ holds. Consequently, $h(\underline{M} \otimes N)=h(M \otimes \underline{N})=1+(l-1) \#(m-1)$. Setting $t=2+(l-1) \#(m-1)=\lambda+\mu+2^{a}+1$, we want to show that $\operatorname{rad}^{t}\left(M^{\prime} \otimes N^{\prime}\right) \subset$ $U \otimes N^{\prime}+M^{\prime} \otimes V$.

From Proposition 5 follows that $h((\operatorname{rad} M) \otimes N)=h(\underline{M} \otimes N)=t-1$ and $h(M \otimes(\operatorname{rad} N))=$ $h(M \otimes \underline{N})=t-1$. Thus, $h(a \otimes b) \leq t-1$ for any $a \otimes b \in \mathcal{B}_{M} \otimes \mathcal{B}_{N} \backslash\{u \otimes v\}$. It remains only to prove that $A_{t}\left(u^{\prime} \otimes v^{\prime}\right)$ and $B_{t}\left(u^{\prime} \otimes v^{\prime}\right)$ lie in $U \otimes N^{\prime}+M^{\prime} \otimes V$. 
The inequality $t>h(\underline{M} \otimes N)$ implies that $\operatorname{rad}^{t}\left(M^{\prime} \otimes N^{\prime}\right) \subset\left(\operatorname{soc} M^{\prime}\right) \otimes N^{\prime}+M^{\prime} \otimes V$, and $t>h(M \otimes \underline{N})$ gives $\operatorname{rad}^{t}\left(M^{\prime} \otimes N^{\prime}\right) \subset U \otimes N^{\prime}+M^{\prime} \otimes\left(\operatorname{soc} N^{\prime}\right)$. Hence

$$
\operatorname{rad}^{t}\left(M^{\prime} \otimes N^{\prime}\right) \subset U \otimes N^{\prime}+M^{\prime} \otimes V+\left(\operatorname{soc} M^{\prime}\right) \otimes\left(\operatorname{soc} N^{\prime}\right)
$$

The restriction of the basis $\mathcal{B}_{M^{\prime}} \otimes \mathcal{B}_{N^{\prime}}$ of $M^{\prime} \otimes N^{\prime}$ to soc $M^{\prime} \otimes \operatorname{soc} N^{\prime}$ is a basis of the latter, and the expansion of $A_{t}\left(u^{\prime} \otimes v^{\prime}\right)$ in $\mathcal{B}_{M^{\prime}} \otimes \mathcal{B}_{N^{\prime}}$ has the form $A_{t} \cdot\left(u^{\prime} \otimes v^{\prime}\right)=z+w$, where

$$
z=\alpha A_{l} u^{\prime} \otimes A_{m} v^{\prime}+\beta A_{l} u^{\prime} \otimes B_{m} v^{\prime}+\gamma B_{l} u^{\prime} \otimes A_{m} v^{\prime} \in \operatorname{soc} M^{\prime} \otimes \operatorname{soc} N^{\prime}
$$

with

$$
\alpha=Q_{t}^{(l, m)}, \quad \beta=Q_{t-1}^{(l-1, m)}, \quad \gamma=Q_{t-1}^{(l, m-1)},
$$

and

$$
w \in \operatorname{span}\left(\left(\mathcal{B}_{M^{\prime}} \otimes \mathcal{B}_{N^{\prime}}\right) \backslash\left(\operatorname{soc} M^{\prime} \otimes \operatorname{soc} N^{\prime}\right)\right) .
$$

By (17), $w \in U \otimes N^{\prime}+M^{\prime} \otimes V$, so to show $A_{t} \cdot\left(u^{\prime} \otimes v^{\prime}\right) \in U \otimes N^{\prime}+M^{\prime} \otimes V$ it is enough to prove that $z \in U \otimes N^{\prime}+M^{\prime} \otimes V$.

Remember that $l=\lambda+2^{a}, m=\mu+2^{a}$, with $\lambda \perp \mu, 2^{a+1} \mid \lambda, \mu$ and $a \geqslant 1$. Setting $j=l+m-t$ we have

$$
\alpha=Q_{t}^{(l, m)} \equiv\left(\begin{array}{c}
t+j \\
l+j
\end{array}\right)\left(\begin{array}{c}
l+j \\
2 j
\end{array}\right)=\left(\begin{array}{c}
\lambda+\mu+2^{a+1} \\
\lambda+2^{a+1}-1
\end{array}\right)\left(\begin{array}{c}
\lambda+2^{a+1}-1 \\
2^{a+1}-2
\end{array}\right) \equiv 0,
$$

since $\lambda+2^{a+1}-1 \equiv 1, \lambda+\mu+2^{a+1} \equiv 0$, implying $\left(\begin{array}{c}\lambda+\mu+2^{a+1} \\ \lambda+2^{a+1}-1\end{array}\right) \equiv 0$,

$$
\begin{aligned}
& \beta=Q_{t-1}^{(l-1, m)}=\left(\begin{array}{c}
t-1+j \\
l-1+j
\end{array}\right)\left(\begin{array}{c}
l-1+j \\
2 j
\end{array}\right)=\left(\begin{array}{c}
\lambda+\mu+2^{a+1}-1 \\
\lambda+2^{a+1}-2
\end{array}\right)\left(\begin{array}{c}
\lambda+2^{a+1}-2 \\
2^{a+1}-2
\end{array}\right) \equiv 1, \\
& \gamma=Q_{t-1}^{(l, m-1)}=\left(\begin{array}{c}
t-1+j \\
l+j
\end{array}\right)\left(\begin{array}{c}
l+j \\
2 j
\end{array}\right)=\left(\begin{array}{c}
\lambda+\mu+2^{a+1}-1 \\
\lambda+2^{a+1}-1
\end{array}\right)\left(\begin{array}{c}
\lambda+2^{a+1}-1 \\
2^{a+1}-2
\end{array}\right) \equiv 1,
\end{aligned}
$$

where Lucas' theorem and the observation that $\left(\begin{array}{c}2^{a+1}-1 \\ 2^{a+1}-2\end{array}\right)=\left(\sum_{\sum_{i=1}^{a} 2^{i}}^{a} 2^{i}\right) \equiv 1$ are used in the two latter calculations. This means that $z=A_{l} u^{\prime} \otimes B_{m} v^{\prime}+B_{l} u^{\prime} \otimes A_{m} v^{\prime} \in U \otimes N^{\prime}+M^{\prime} \otimes V$, proving that $A_{t} \cdot\left(u^{\prime} \otimes v^{\prime}\right) \in U \otimes N^{\prime}+M^{\prime} \otimes V$ and hence $A_{t} \cdot(u \otimes v)=0$.

Finally,

$$
B_{t} \cdot\left(u^{\prime} \otimes v^{\prime}\right)=\alpha B_{l} u^{\prime} \otimes B_{m} v^{\prime}+\beta B_{l} u^{\prime} \otimes A_{m} v^{\prime}+\gamma A_{l} u^{\prime} \otimes B_{m} v^{\prime}+w^{\prime}
$$

where $w^{\prime} \in \operatorname{span}\left(\left(\mathcal{B}_{M^{\prime}} \otimes \mathcal{B}_{N^{\prime}}\right) \backslash\left(\operatorname{soc} M^{\prime} \otimes \operatorname{soc} N^{\prime}\right)\right)$. Again, Equation (17) gives $w^{\prime} \in U \otimes N^{\prime}+$ $M^{\prime} \otimes V$, and with (18)-(20) we conclude that

$$
B_{t} \cdot\left(u^{\prime} \otimes v^{\prime}\right)=B_{l} u^{\prime} \otimes A_{m} v^{\prime}+A_{l} u^{\prime} \otimes B_{m} v^{\prime}+w^{\prime} \in U \otimes N^{\prime}+M^{\prime} \otimes V .
$$

This proves that $\operatorname{rad}^{t}\left(M^{\prime} \otimes N^{\prime}\right) \subset U \otimes N^{\prime}+M^{\prime} \otimes V$ and hence $h(M \otimes N)=t-1=1+(l-$ 1)\#(m-1).

\section{ACKNOWLEDGEMENTS}

The work for this article was partly carried out at the Mathematical Institute, University of Oxford. The authors wish to thank Karin Erdmann for her encouragement and support.

\section{REFERENCES}

[1] L. Archer. On certain quotients of the Green rings of dihedral 2-groups. J. Pure Appl. Algebra, 212(8):18881897, 2008.

[2] Michael J. J. Barry. Decomposing tensor products and exterior and symmetric squares. J. Group Theory, 14(1):59-82, 2011.

[3] D. J. Benson and J. F. Carlson. Nilpotent elements in the Green ring. J. Algebra, 104(2):329-350, 1986.

[4] Christine Bessenrodt. Endotrivial modules and the Auslander-Reiten quiver. In Representation theory of finite groups and finite-dimensional algebras (Bielefeld, 1991), volume 95 of Progr. Math., pages 317-326. Birkhäuser, 1991.

[5] S. B. Conlon. Certain representation algebras. J. Austral. Math. Soc., 5:83-99, 1965. 
[6] J. A. Green. The modular representation algebra of a finite group. Illinois J. Math, 6:607-619, 1962.

[7] K. Iima and R. Iwamatsu. On the Jordan decomposition of tensored matrices of Jordan canonical forms. Math. J. Okayama Univ., 51:133-148, 2009.

[8] E. Lucas. Sur les congruences des nombres eulriens et les coefficients diffrentiels des functions trigonomtriques suivant un module premier. Bull. Soc. Math. France, 6:49-54, 1878.

[9] C. W. Norman. On Jordan bases for the tensor product and Kronecker sum and their elementary divisors over fields of prime characteristic. Linear Multilinear Algebra, 56(4):415-451, 2008.

[10] C. M. Ringel. The indecomposable representations of the dihedral 2-groups. Math. Ann., 214:19-34, 1975.

[11] Bhama Srinivasan. The modular representation ring of a cyclic p-group. Proc. London Math. Soc. (3), 14:677688, 1964.

[12] Richard P. Stanley. Enumerative combinatorics. Vol. 1, volume 49 of Cambridge Studies in Advanced Mathematics. Cambridge University Press, 1997.

Darpö: Graduate School of Mathematics, Nagoya University, Furocho, Chikusaku, Nagoya, Japan

E-mail address: darpo@math.nagoya-u.ac.jp

Gill: Department of Algebra, Charles University, Sokolovska 83, Praha 8, 186 75, Czech Republic

E-mail address: gill@karlin.mff.cuni.cz 